\title{
Frequency and diagnostic reliability of laboratory variables in cows with traumatic reticuloperitonitis and type 1,2,3,4 and 5 abomasal ulcer
}

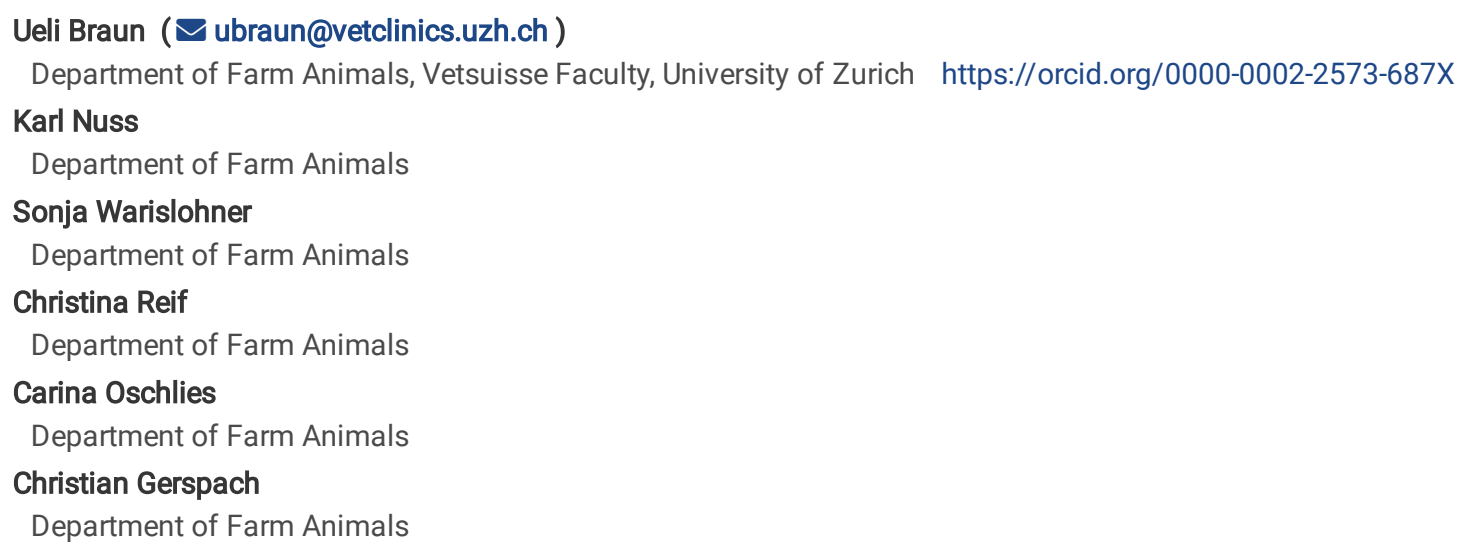

Research article

Keywords: Cattle, traumatic reticuloperitonitis, abomasal ulcus, sensitivity, specificity, predictive values, positive likelihood ratio+

Posted Date: October 26th, 2020

DOI: https://doi.org/10.21203/rs.3.rs-96358/v1

License: (c) (i) This work is licensed under a Creative Commons Attribution 4.0 International License. Read Full License 


\section{Abstract}

Background: A number of laboratory abnormalities occur in cows with traumatic reticuloperitonitis (TRP) as well as in those with abomasal ulcers (AU). This prompted us to compare the frequencies of laboratory abnormalities of healthy cows and cows with TRP and cows with abomasal ulcers and to calculate diagnostic sensitivities and specificities, predictive values and positive likelihood ratios for laboratory findings. The study included 182 healthy control cows, 503 cows with TRP, 94 cows with U1, 145 cows with U2, 60 cows with U3, 87 cows with U4 and 14 cows with U5. Haematocrit, total leukocyte count, concentrations of total protein, fibrinogen, urea, potassium and chloride, base excess and rumen chloride concentration were analysed.

Results: Values outside the reference interval occurred in 2 to $24 \%$ of control cows (rumen chloride $2 \%$, urea $6 \%$, serum chloride $11 \%$, haematocrit $13 \%$, base excess $18 \%$, fibrinogen $20 \%$, total protein $21 \%$, total leukocyte count $22 \%$ and potassium $24 \%$, which made differentiation of healthy and ill cows difficult. Therefore, the variables best suited for distinguishing healthy and ill cows were rumen chloride and blood urea concentration. This was also supported by an $\mathrm{LR}_{+}$of 14 to 27 for rumen chloride $>30 \mathrm{mmol} / \mathrm{l}$ and 6 to 15 for blood urea $>6.5 \mathrm{mmol} / \mathrm{l}$ in cows with ulcers. Urea also had a high diagnostic specificity and like rumen chloride was suited for differentiation of healthy and diseased cows. The urea concentration was $>8.5$ $\mathrm{mmol} / \mathrm{l}$ in only $0.5 \%$ of controls, and the $\mathrm{LR}_{+}$for a urea concentration $>8.5 \mathrm{mmol} / \mathrm{I}$ ranged from 11 in cows with TRP to 128 in cows with U2. Except for cows with TRP, azotaemia was significantly more frequent in ill cows than in controls. Cows with U2 (70\%) had urea concentrations $>8.5$ significantly more often than cows of the other groups, which may have been prerenal azotaemia attributable to hypovolaemia. Even though the groups of ill cows differed significantly with respect to several variables, no variables were identified to reliably differentiate the various groups.

Conclusions: Isolated results are not suitable to distinguish among groups of ill cows and instead the history, the clinical findings and results of additional diagnostic techniques such as ultrasonography are required.

\section{Background}

Traumatic reticuloperitonitis (TRP) and abomasal ulcers are important intestinal disorders of cattle [1, 2]. Abomasal ulcers are classified as type 1 (U1), 2 (U2), 3 (U3), 4 (U4) or 5 (U5) [3-7]. Type 1 ulcer is non-perforating and accompanied by minimal haemorrhage, and is subdivided into subtypes 1a, 1b, 1c and 1d [8, 9]. Type 2 ulcer is characterised by the erosion of a large blood vessel causing intraluminal haemorrhage and anaemia. Type 3 ulcer is perforating and accompanied by localised peritonitis, U4 is perforating and accompanied by generalised peritonitis and U5 has perforated into the omental bursa. Laboratory findings in cows with TRP [10], U1 [11], U2 [12], U3 [13], U4 [14] and U5 [7] were recently described in detail. Laboratory findings reported in older studies of cows with TRP and abomasal ulcer have also been discussed [7, 10]. Of 94 cows with U1, the majority had hypokalaemia (68\%), positive base excess (60\%) and azotaemia (51\%) [11], and of 145 cows with U2, the most frequent laboratory findings were azotaemia (89\%), low haematocrit (82\%), hypokalaemia (81\%), hypoproteinaemia (74\%) and metabolic acidosis (61\%) [12]. The only laboratory abnormality that was commonly seen in 60 cows with U3 was hypokalaemia (75\%) [13]. Haemoconcentration (69\%) and azotaemia (56\%) [14] were the main abnormalities in 87 cows with U4, and hypokalaemia (71\%), haemoconcentration (57\%) and metabolic acidosis (57\%) were the most common abnormalities in 14 cows with U5 [7]. Hyperfibrinogenaemia (69\%) and hyperproteinaemia (64\%) were the major laboratory abnormalities in 503 cows with TRP [10]. The degree of increase in rumen chloride concentration also varied among the different diseases. The abovementioned studies showed that hypokalaemia and azotaemia are common to all groups, whereas haemoconcentration is typically seen in cows with $\mathrm{U} 4$ and $\mathrm{U}$, haemodilution in cows with $\mathrm{U} 2$, hyperproteinaemia in cows with TRP and hypoproteinaemia in cows with U2. Furthermore, the frequency with which the laboratory abnormalities occur varied greatly among the groups. The prognosis also differed significantly and was usually good in cows with TRP, often fair in cows with U2, but generally poor in cows with U4 and U5. Finally, TRP and abomasal ulcer require different therapeutic approaches. The diagnostic reliability of clinical signs in cows with TPR and abomasal ulcers was recently investigated [15]. From a diagnostic standpoint, it would be desirable to use also laboratory variables to aid in differentiation of cows with TRP and various types of abomasal ulcers. To investigate this, the frequencies of laboratory abnormalities recorded in cows with TRP and abomasal ulcer were compared and the parameters diagnostic sensitivity and specificity, predictive values and positive likelihood ratio $\left(\mathrm{LR}_{+}\right)$calculated.

\section{Methods}

\section{Animals}

A total of 1,085 cows including 182 healthy controls, 503 cows with TRP, 94 cows with U1, 145 cows with U2, 60 cows with U3, 87 cows with U4 and 14 cows with U5 were used. All animals were privately owned and transported to the Veterinary Teaching Hospital of the University of Zurich for clinical examination. The sample size differed between groups and was dictated by the case load, which varied depending of the incidence of the disease in the population. The frequency and diagnostic reliability of the clinical findings of these cows were the subject of a recent publication [15].

\section{Tentative and definitive diagnoses of TRP and type 1, 2, 3, 4 or 5 abomasal ulcer, treatment and response to treatment}

The criteria for tentative and confirmed diagnoses of TRP and abomasal ulcers, inclusion and exclusion criteria, and treatment and response to treatment of the cows in this study were recently published [15]. 


\section{Euthanasia}

Euthanasia was done with pentobarbital (Esconarkon, Streuli Pharma), $80 \mathrm{mg} / \mathrm{kg}$ body weight intravenously.

\section{Analysis of laboratory variables}

The laboratory findings of the controls [16] and the cows with TRP [10], U1 [11], U2 [12], U3 [13], U4 [14] and U5 [7] have been described in detail. The variables haematocrit, total leukocyte count, concentrations of total protein, fibrinogen, urea, potassium and chloride, base excess and rumen chloride concentration were analysed. The reference intervals for total leukocyte count $(5,000-10,000 \mathrm{cells} / \mu \mathrm{l})$ and fibrinogen $(4-7 \mathrm{~g} / \mathrm{l})$, urea $(2.0-$ $6.5 \mathrm{mmol} / \mathrm{l})$ and potassium concentrations (4-5 mmol/l) have been published previously [10, 12-14, 17]. The reference interval for the haematocrit

of $30-35 \%$ [10,12-14.17] was modified to $27-37 \%$ because otherwise $41 \%$ of the control cows would have had a value outside the reference interval. For similar reasons, the reference interval for total protein concentration was expanded from $60-80 \mathrm{~g} / \mathrm{l}[10,12-14,17]$ to $60-85 \mathrm{~g} / \mathrm{l}$, serum chloride concentration from 96-105 mmol/l $[13,17]$ to $91-105 \mathrm{mmol} / \mathrm{l}$, base excess from -2 to $+2[10,12-14,17]$ to -4 to $+4 \mathrm{mmol} / \mathrm{l}$ and rumen chloride concentration from $15-30[18]$ to $6-30 \mathrm{mmol} / \mathrm{l}$.

\section{Statistical analysis}

The program SPSS Version 25 was used for analysis. The Shapiro-Wilk test showed that only total protein and urea concentrations had normal distribution and therefor the medians and 25th and 75th percentiles were calculated. The medians underwent one-factor analysis of variance and pair-wise comparison using the Kruskal-Wallis test, and frequency distributions of all variables were calculated for the controls and six disease groups. Laboratory results were arbitrarily divided into appropriate ranges (see Table 2, example haematocrit; $27-37 \%, \leq 20$ and $>20 \%,<27$ and $\geq$ $27 \%, \leq 37$ and $>37 \%, \leq 44$ and $>44 \%$ ), and the ranges compared among groups using the chi-square and the Bonferroni post-hoc tests. A P-value < 0.05 was considered significant. For each variable, the diagnostic sensitivity $(a /[a+c])$, diagnostic specificity $(d /[b+d])$, positive likelihood ratio $\left(L R_{+}\right.$, sensitivity/[1-specificity]), positive predictive value $(a /[a+b])$ and negative predictive value $(d /[c+d])$ were calculated (a, true positive; $b$, false positive; $c$, false negative; $d$, true negative). A false positive result was an abnormal finding in a control cow and a false negative result was a normal finding in a cow with TRP or abomasal ulcer. The occurrence of a decreased (<27\%), normal (27-37\%) and elevated ( $>37 \%)$ haematocrit accompanied by decreased ( $<60 \mathrm{~g} / \mathrm{l})$, normal $(60-85 \mathrm{~g} / \mathrm{l})$ and elevated total protein concentration $(>85 \mathrm{~g} / \mathrm{l})$, and the occurrence of decreased, normal and elevated total protein concentration accompanied by decreased $(<4 \mathrm{~g} / \mathrm{l})$, normal $(4-7 \mathrm{~g} / \mathrm{l})$ and elevated $(>7 \mathrm{~g} / \mathrm{l})$ fibrinogen concentration were investigated. 
Table 2

Frequency distributions of the laboratory findings in healthy control cows and in cows with traumatic reticuloperitonitis (TRP), abomasal ulcer type 1

\begin{tabular}{|c|c|c|c|c|c|c|c|c|c|}
\hline Variable & Classification & Controls & TRP & U1 & $\mathrm{U} 2$ & U3 & U4 & U5 & $\mathrm{Chi}^{2}$ \\
\hline $\begin{array}{l}\text { Haematocrit } \\
\text { (normal) }\end{array}$ & $\begin{array}{l}27-37 \% \\
<27 \text { or }>37 \%\end{array}$ & $\begin{array}{l}159 \\
(87 \%)^{2,3,4,5,6,7} \\
23(13 \%)\end{array}$ & $\begin{array}{l}378 \\
(75 \%)^{1,3,4,6.7} \\
123(25 \%)\end{array}$ & $\begin{array}{l}54 \\
(57 \%)^{1,2,4,6} \\
40(43 \%)\end{array}$ & $\begin{array}{l}21 \\
(15 \%)^{1,2,3,5} \\
124(85 \%)\end{array}$ & $\begin{array}{l}41 \\
(68 \%)^{1,4,6}\end{array}$ & $\begin{array}{l}26 \\
(30 \%)^{1,2,3,5}\end{array}$ & $\begin{array}{l}5(36 \%)^{1,2} \\
9(64 \%)\end{array}$ & 274 \\
\hline \multirow[t]{3}{*}{$\begin{array}{l}\text { Haematocrit } \\
\text { decreased }\end{array}$} & $\begin{array}{l}<27 \% \\
\geq 27 \%\end{array}$ & $\begin{array}{l}7(4 \%)^{2,4} \\
175(96 \%)\end{array}$ & $\begin{array}{l}86(17 \%)^{1,4} \\
415(83 \%)\end{array}$ & $\begin{array}{l}7(7 \%)^{4} \\
87(93 \%)\end{array}$ & $\begin{array}{l}115 \\
(79 \%)^{1,2,3,5,6,7} \\
30(21 \%)\end{array}$ & $\begin{array}{l}3(5 \%)^{4} \\
57(95 \%)\end{array}$ & $\begin{array}{l}6(7 \%)^{4} \\
81(93 \%)\end{array}$ & $\begin{array}{l}2(14 \%)^{4} \\
12(86 \%)\end{array}$ & 366 \\
\hline & $\leq 20 \%$ & $0(0 \%)^{4,7}$ & $7(1 \%)^{4}$ & $0(0 \%)^{4}$ & $\begin{array}{l}102 \\
(70 \%)^{1,2,3,5,6,7}\end{array}$ & $1(2 \%)^{4}$ & $4(5 \%)^{4}$ & $1(7 \%)^{1,4}$ & \\
\hline & $>20 \%$ & $182(100 \%)$ & 494 (99\%) & 94 (100\%) & $43(30 \%)$ & $59(98 \%)$ & $83(17 \%)$ & $13(93 \%)$ & 631 \\
\hline \multirow[t]{2}{*}{$\begin{array}{l}\text { Haematocrit } \\
\text { increased }\end{array}$} & $\begin{array}{l}>37 \% \\
\leq 37 \%\end{array}$ & $\begin{array}{l}16(9 \%)^{3,5,6,7} \\
166(91 \%)\end{array}$ & $\begin{array}{l}37(7 \%)^{3,5,6,7} \\
464(93 \%)\end{array}$ & $\begin{array}{l}33 \\
(35 \%)^{1,2,4,6} \\
61(65 \%)\end{array}$ & $\begin{array}{l}9(6 \%)^{3,5,6,7} \\
136(94 \%)\end{array}$ & $\begin{array}{l}16 \\
(27 \%)^{1,2,4,6} \\
44(73 \%)\end{array}$ & $\begin{array}{l}55 \\
(63 \%)^{1,2,3,4,5} \\
32(37 \%)\end{array}$ & $\begin{array}{l}7 \\
(50 \%)^{1,2,4} \\
7(50 \%)\end{array}$ & 232 \\
\hline & $\leq 44 \%$ & $182(100 \%)$ & 494 (99\%) & $3(3 \%)^{6}$ & $144(99 \%)$ & $3(5 \%)^{6}$ & $\begin{array}{l}29 \\
(33 \%)^{1,2,3,4,5} \\
58(67 \%)\end{array}$ & $1(7 \%)^{1}$ & 213 \\
\hline $\begin{array}{l}\text { Total } \\
\text { leukocyte } \\
\text { count } \\
\text { (normal) }\end{array}$ & $\begin{array}{l}5000- \\
10^{\prime} 000 / \mu l \\
<5000 \text { or > } \\
10^{\prime} 000 / \mu l\end{array}$ & $\begin{array}{l}141(78 \%)^{2,4,6} \\
41(22 \%)\end{array}$ & $\begin{array}{l}272(54 \%)^{1} \\
229(46 \%)\end{array}$ & $\begin{array}{l}58(62 \%) \\
36(38 \%)\end{array}$ & $\begin{array}{l}78(55 \%)^{1} \\
65(45 \%)\end{array}$ & $\begin{array}{l}38(63 \%) \\
22(37 \%)\end{array}$ & $\begin{array}{l}40(47 \%)^{1} \\
46(53 \%)\end{array}$ & $\begin{array}{l}10(71 \%) \\
4(29 \%)\end{array}$ & 39 \\
\hline $\begin{array}{l}\text { Total } \\
\text { leukocyte } \\
\text { count }\end{array}$ & $<5000 / \mu l$ & $7(4 \%)^{6}$ & $20(4 \%)^{3,4,6}$ & $\begin{array}{l}12 \\
(13 \%)^{2,6}\end{array}$ & $17(12 \%)^{2,6}$ & $7(12 \%)^{6}$ & $\begin{array}{l}30 \\
(35 \%)^{1,2,3,4,5}\end{array}$ & $2(14 \%)$ & \\
\hline \multirow[t]{3}{*}{ decreased } & $\geq 5000 / \mu \mathrm{l}$ & $175(96 \%)$ & 481 (96\%) & $82(87 \%)$ & $126(88 \%)$ & $53(88 \%)$ & $56(65 \%)$ & $12(86 \%)$ & 98 \\
\hline & $<2500 / \mu l$ & $1(1 \%)$ & $1(0.2 \%)^{6}$ & $2(2 \%)$ & $1(1 \%)$ & $0(0 \%)$ & $4(5 \%)^{2}$ & $0(0 \%)$ & \\
\hline & $\geq 2500 / \mu \mathrm{l}$ & 181 (99\%) & 500 (99.8\%) & $92(98 \%)$ & 142 (99\%) & 60 (100\%) & 82 (95\%) & $14(100 \%)$ & 20 \\
\hline
\end{tabular}

\begin{tabular}{|l|}
\hline${ }^{1}$ Different from controls $\mathrm{P}<0.01$ \\
\hline${ }^{2}$ Different from TRP $\mathrm{P}<0.01$ \\
\hline${ }^{3}$ Different from U1 $\mathrm{P}<0.01$ \\
\hline${ }^{4}$ Different from U2 $\mathrm{P}<0.01$ \\
\hline${ }^{5}$ Different from U3 $\mathrm{P}<0.01$ \\
\hline${ }^{6}$ Different from U4 $\mathrm{P}<0.01$ \\
\hline${ }^{7}$ Different from U5 $\mathrm{P}<0.01$ \\
\hline
\end{tabular}




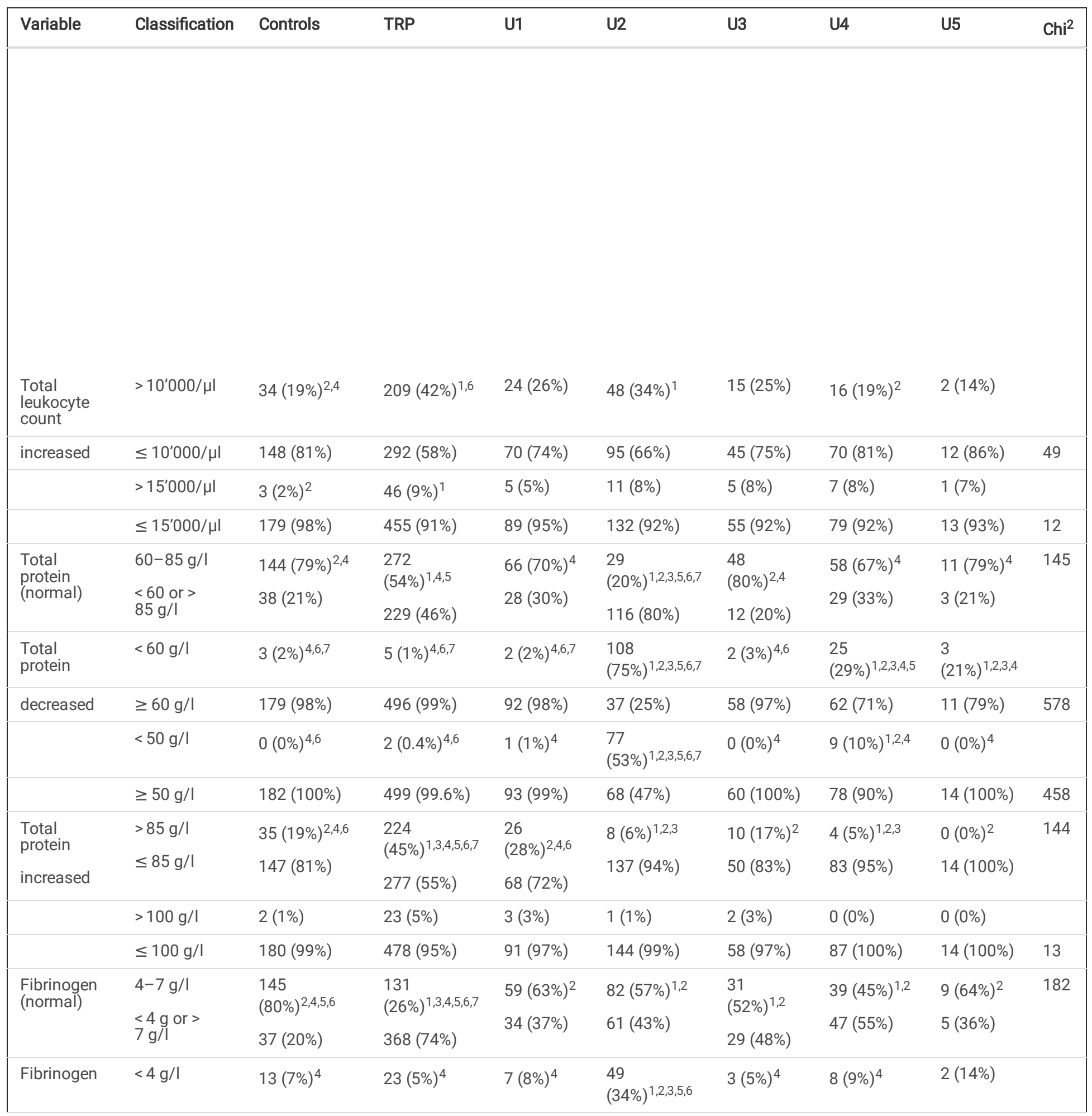

\begin{tabular}{|l||}
\hline${ }^{1}$ Different from controls $P<0.01$ \\
\hline${ }^{2}$ Different from TRP $P<0.01$ \\
\hline${ }^{3}$ Different from U1 $P<0.01$ \\
\hline${ }^{4}$ Different from U2 $P<0.01$ \\
\hline${ }^{5}$ Different from U3 $P<0.01$ \\
\hline${ }^{6}$ Different from U4 $P<0.01$ \\
\hline${ }^{7}$ Different from U5 $P<0.01$ \\
\hline
\end{tabular}




\begin{tabular}{|c|c|c|c|c|c|c|c|c|c|}
\hline Variable & Classification & Controls & TRP & U1 & U2 & U3 & U4 & U5 & $\mathrm{Chi}^{2}$ \\
\hline \multirow[t]{3}{*}{ decreased } & $\geq 4 \mathrm{~g} / \mathrm{l}$ & $169(93 \%)$ & $476(95 \%)$ & $86(92 \%)$ & $94(66 \%)$ & 57 (95\%) & 78 (91\%) & $12(86 \%)$ & 116 \\
\hline & $<3 \mathrm{~g} / \mathrm{l}$ & $7(4 \%)^{4}$ & $15(3 \%)^{4}$ & $6(7 \%)^{4}$ & $\begin{array}{l}32 \\
(22 \%)^{1,2,3,6}\end{array}$ & $3(5 \%)$ & $3(4 \%)^{4}$ & $2(14 \%)^{1,4}$ & \\
\hline & $\geq 3 \mathrm{~g} / \mathrm{l}$ & $175(96 \%)$ & $484(97 \%)$ & 87 (93\%) & $111(78 \%)$ & $57(95 \%$ & $83(96 \%)$ & $12(86 \%)$ & 76 \\
\hline Fibrinogen & $>7 \mathrm{~g} / \mathrm{l}$ & $24(13 \%)^{2,3,5,6}$ & $\begin{array}{l}345 \\
(69 \%)^{1,3,4,5,6,7}\end{array}$ & $\begin{array}{l}27 \\
(29 \%)^{1,2,4}\end{array}$ & $12(8 \%)^{2,3,5,6}$ & $\begin{array}{l}26 \\
(43 \%)^{1,2,4}\end{array}$ & $\begin{array}{l}39 \\
(45 \%)^{1,2,4}\end{array}$ & $3(21 \%)^{2}$ & \\
\hline \multirow[t]{3}{*}{ increased } & $\leq 7 \mathrm{~g} / \mathrm{l}$ & $158(87 \%)$ & $154(31 \%)$ & $66(71 \%)$ & 131 (92\%) & 34 (57\%) & 47 (55\%) & $11(79 \%)$ & 283 \\
\hline & $>9 \mathrm{~g} / \mathrm{l}$ & $3(2 \%)^{2,3,5,6}$ & $\begin{array}{l}224 \\
(45 \%)^{1,3,4,5,6,7}\end{array}$ & $\begin{array}{l}10 \\
(11 \%)^{1,2}\end{array}$ & $7(5 \%)^{2,5}$ & $\begin{array}{l}14 \\
(23 \%)^{1,2,4}\end{array}$ & $14(16 \%)^{1,2}$ & $0(0 \%)^{2}$ & \\
\hline & $\leq 9 \mathrm{~g} / \mathrm{l}$ & $179(98 \%)$ & $275(55 \%)$ & $83(89 \%)$ & $136(95 \%)$ & $46(77 \%)$ & $72(84 \%)$ & $14(100 \%)$ & 206 \\
\hline \multirow[t]{2}{*}{$\begin{array}{l}\text { Urea } \\
\text { (normal) }\end{array}$} & $\begin{array}{l}2- \\
6.5 \mathrm{mmol} / \mathrm{l}\end{array}$ & $\begin{array}{l}171 \\
(94 \%)^{3,4,5,6,7}\end{array}$ & $\begin{array}{l}431 \\
(86 \%)^{3,4,5,6}\end{array}$ & $\begin{array}{l}46 \\
(49 \%)^{1,2,4}\end{array}$ & $\begin{array}{l}16 \\
(11 \%)^{1,2,3,5,6,7}\end{array}$ & $\begin{array}{l}39 \\
(65 \%)^{1,2,4}\end{array}$ & $\begin{array}{l}38 \\
(44 \%)^{1,2,4}\end{array}$ & \multirow{2}{*}{$\begin{array}{l}8(57 \%)^{1,4} \\
6(43 \%)\end{array}$} & \multirow[t]{2}{*}{392} \\
\hline & $>6.5 \mathrm{mmol} / \mathrm{l}$ & $11(6 \%)$ & $70(14 \%)$ & $48(51 \%)$ & $128(89 \%)$ & $21(35 \%)$ & 48 (56\%) & & \\
\hline Urea & $>6.5 \mathrm{mmol} / \mathrm{l}$ & $11(6 \%)^{3,4,5,6,7}$ & $\begin{array}{l}70 \\
(14 \%)^{3,4,5,6}\end{array}$ & $\begin{array}{l}48 \\
(51 \%)^{1,2,4}\end{array}$ & $\begin{array}{l}128 \\
(89 \%)^{1,2,3,5,6,7}\end{array}$ & $\begin{array}{l}21 \\
(35 \%)^{1,2,4}\end{array}$ & $\begin{array}{l}48 \\
(56 \%)^{1,2,4}\end{array}$ & $6(43 \%)^{1,4}$ & \\
\hline \multirow[t]{3}{*}{ increased } & $\leq 6.5 \mathrm{mmol} / \mathrm{l}$ & $171(94 \%)$ & $431(86 \%)$ & $46(49 \%)$ & $16(11 \%)$ & $39(65 \%)$ & $38(44 \%)$ & $8(57 \%)$ & 392 \\
\hline & $>8.5 \mathrm{mmol} / \mathrm{l}$ & $\begin{array}{l}1 \\
(0.5 \%)^{3,4,5,6,7}\end{array}$ & $30(6 \%)^{3,4,6,7}$ & $\begin{array}{l}26 \\
(28 \%)^{1,2,4}\end{array}$ & $\begin{array}{l}101 \\
(70 \%)^{1,2,3,5,6,7}\end{array}$ & $\begin{array}{l}8 \\
(13 \%)^{1,4,6}\end{array}$ & $\begin{array}{l}35 \\
(41 \%)^{1,2,4,5}\end{array}$ & $\begin{array}{l}4 \\
(29 \%)^{1,2,4}\end{array}$ & \\
\hline & $\leq 8.5 \mathrm{mmol} / \mathrm{l}$ & $182(99.5 \%)$ & 471 (94\%) & $68(72 \%)$ & $43(30 \%)$ & $52(87 \%)$ & $51(59 \%)$ & $10(71 \%)$ & 374 \\
\hline \multirow{2}{*}{$\begin{array}{l}\text { Potassium } \\
\text { (normal) }\end{array}$} & $4-5 \mathrm{mmol} / \mathrm{l}$ & $139(76 \%)$ & $132(26 \%)^{1}$ & $27(29 \%)^{1}$ & $25(18 \%)^{1}$ & $15(25 \%)^{1}$ & $17(20 \%)^{1}$ & $3(21 \%)^{1}$ & \multirow[t]{2}{*}{190} \\
\hline & $\begin{array}{l}<4 \text { or }> \\
5 \mathrm{mmol} / \mathrm{l}\end{array}$ & $43(24 \%)$ & $368(74 \%)$ & $67(71 \%)$ & $118(83 \%)$ & $45(75 \%)$ & $69(80 \%)$ & $11(79 \%)$ & \\
\hline Potassium & $<4 \mathrm{mmol} / \mathrm{l}$ & $\begin{array}{l}42 \\
(23 \%)^{2,3,4,5,6,7}\end{array}$ & $361(72 \%)^{1}$ & $64(68 \%)^{1}$ & $116(81 \%)^{1}$ & $45(75 \%)^{1}$ & $62(72 \%)^{1}$ & $10(71 \%)^{1}$ & \\
\hline \multirow[t]{3}{*}{ decreased } & $\geq 4 \mathrm{mmol} / \mathrm{l}$ & $140(77 \%)$ & $139(28 \%)$ & 30 (32\%) & 27 (19\%) & $15(25 \%)$ & $24(28 \%)$ & $4(29 \%)$ & 173 \\
\hline & $<3 \mathrm{mmol} / \mathrm{l}$ & $\begin{array}{l}1 \\
(0.5 \%)^{2,3,4,5,6,7}\end{array}$ & $63(13 \%)^{1}$ & $20(21 \%)^{1}$ & $29(20 \%)^{1}$ & $15(25 \%)^{1}$ & $17(20 \%)^{1}$ & $2(14 \%)^{1}$ & \\
\hline & $\geq 3 \mathrm{mmol} / \mathrm{l}$ & 181 (99.5\%) & 437 (87\%) & 74 (79\%) & $114(80 \%)$ & 45 (75\%) & $69(80 \%)$ & 12 (86\%) & 46 \\
\hline \multirow[t]{2}{*}{$\begin{array}{l}\text { Chloride } \\
\text { (normal) }\end{array}$} & $\begin{array}{l}91- \\
105 \mathrm{mmol} / \mathrm{l}\end{array}$ & \multirow{2}{*}{$\begin{array}{l}161(89 \%)^{3,4,5} \\
21(11 \%)\end{array}$} & \multirow{2}{*}{$\begin{array}{l}405 \\
(81 \%)^{3,4,5} \\
94(19 \%)\end{array}$} & \multirow{2}{*}{$\begin{array}{l}49 \\
(52 \%)^{1,2,6} \\
45(48 \%)\end{array}$} & \multirow{2}{*}{$\begin{array}{l}91(64 \%)^{1,2} \\
51(36 \%)\end{array}$} & \multirow{2}{*}{$\begin{array}{l}34 \\
(58 \%)^{1,2} \\
25(42 \%)\end{array}$} & $64(74 \%)^{3}$ & 10 (71\%) & \multirow[t]{2}{*}{74} \\
\hline & $\begin{array}{l}\leq 90 \text { or }> \\
105 \mathrm{mmol} / \mathrm{l}\end{array}$ & & & & & & $22(26 \%)$ & $4(29 \%)$ & \\
\hline Chloride & $\leq 90 \mathrm{mmol} / \mathrm{l}$ & $4(2 \%)^{3,5,6}$ & $36(7 \%)^{3,5,6}$ & $\begin{array}{l}17 \\
(18 \%)^{1,2}\end{array}$ & $11(8 \%)$ & $\begin{array}{l}13 \\
(22 \%)^{1,2}\end{array}$ & $17(20 \%)^{1,2}$ & $2(14 \%)$ & \\
\hline \multirow[t]{2}{*}{ decreased } & $>90 \mathrm{mmol} / \mathrm{l}$ & 178 (98\%) & $463(93 \%)$ & 77 (82\%) & 131 (92\%) & 46 (78\%) & 69 (80\%) & $12(86 \%)$ & 45 \\
\hline & $\leq 80 \mathrm{mmol} / \mathrm{l}$ & $2(1 \%)^{3}$ & $8(2 \%)^{3}$ & $8(9 \%)^{1,2}$ & $2(1 \%)$ & $2(3 \%)$ & $4(5 \%)$ & $1(7 \%)$ & \\
\hline
\end{tabular}

\begin{tabular}{|l||}
\hline${ }^{1}$ Different from controls $P<0.01$ \\
\hline${ }^{2}$ Different from TRP $P<0.01$ \\
\hline${ }^{3}$ Different from U1 $P<0.01$ \\
\hline${ }^{4}$ Different from U2 $P<0.01$ \\
\hline${ }^{5}$ Different from U3 $P<0.01$ \\
\hline${ }^{6}$ Different from U4 $P<0.01$ \\
\hline${ }^{7}$ Different from U5 $P<0.01$ \\
\hline
\end{tabular}




\begin{tabular}{|c|c|c|c|c|c|c|c|c|c|}
\hline Variable & Classification & Controls & TRP & U1 & U2 & U3 & U4 & U5 & $\mathrm{Chi}^{2}$ \\
\hline & $>80 \mathrm{mmol} / \mathrm{l}$ & 180 (99\%) & 491 (98\%) & $86(91 \%)$ & 140 (99\%) & 57 (97\%) & $82(95 \%)$ & $13(93 \%)$ & 21 \\
\hline \multirow{2}{*}{$\begin{array}{l}\text { Chloride } \\
\text { increased }\end{array}$} & $>105 \mathrm{mmol} / \mathrm{l}$ & $17(9 \%)^{3,4}$ & $58(12 \%)^{3,4}$ & \multirow{2}{*}{$\begin{array}{l}28 \\
(30 \%)^{1,2,6}\end{array}$} & $40(28 \%)^{1,2,6}$ & $12(20 \%)$ & $5(6 \%)^{3,4}$ & $2(14 \%)$ & \multirow[t]{2}{*}{51} \\
\hline & $\leq$ & 165 (91\%) & $440(88 \%)$ & & $102(72 \%)$ & $47(80 \%)$ & $81(94 \%)$ & $12(86 \%)$ & \\
\hline \multirow{2}{*}{$\begin{array}{l}\text { Base excess } \\
\text { (normal) }\end{array}$} & $\begin{array}{l}-4 \text { to }+ \\
4 \mathrm{mmol} / 1\end{array}$ & 145 & \multirow{2}{*}{$\begin{array}{l}254(55 \%)^{1} \\
210(45 \%)\end{array}$} & \multirow{2}{*}{$\begin{array}{l}39(44 \%)^{1} \\
49(56 \%)\end{array}$} & \multirow{2}{*}{$\begin{array}{l}61(52 \%)^{1} \\
56(48 \%)\end{array}$} & \multirow{2}{*}{$\begin{array}{l}32(57 \%)^{1} \\
24(43 \%)\end{array}$} & \multirow{2}{*}{$\begin{array}{l}34(47 \%)^{1} \\
38(53 \%)\end{array}$} & \multirow{2}{*}{$\begin{array}{l}9(64 \%) \\
5(36 \%)\end{array}$} & \multirow[t]{2}{*}{57} \\
\hline & $\begin{array}{l}<-4 \text { or }> \\
+4 \mathrm{mmol} / /\end{array}$ & $31(18 \%)$ & & & & & & & \\
\hline Base excess & $<-4 \mathrm{mmol} / \mathrm{l}$ & $10(6 \%)^{4,6}$ & $26(6 \%)^{4,6}$ & $11(13 \%)$ & $25(21 \%)^{1,2}$ & $3(5 \%)$ & $13(18 \%)^{1,2}$ & $2(14 \%)$ & \\
\hline \multirow[t]{3}{*}{ decreased } & $\geq-4 \mathrm{mmol} / \mathrm{l}$ & $166(94 \%)$ & 438 (94\%) & 77 (87\%) & 92 (79\%) & 53 (95\%) & $59(82 \%)$ & $12(86 \%)$ & 36 \\
\hline & $<-8 \mathrm{mmol} / \mathrm{l}$ & $1(0.5 \%)^{4}$ & $2(0.4 \%)^{4,6,7}$ & $3(3 \%)$ & $13(11 \%)^{1,2}$ & $0(0 \%)$ & $4(6 \%)^{2}$ & $1(7 \%)^{2}$ & \\
\hline & $\geq-8 \mathrm{mmol} / \mathrm{l}$ & 175 (99.5\%) & $462(99.6 \%)$ & 85 (97\%) & 104 (89\%) & $56(100 \%)$ & $68(94 \%)$ & $13(93 \%)$ & 55 \\
\hline Base excess & $>+4 \mathrm{mmol} / \mathrm{l}$ & $\begin{array}{l}21 \\
(12 \%)^{2,3,4,5,6}\end{array}$ & $181(39 \%)^{1}$ & $38(43 \%)^{1}$ & $31(27 \%)^{1}$ & $21(38 \%)^{1}$ & $25(35 \%)^{1}$ & $3(21 \%)$ & \\
\hline \multirow[t]{3}{*}{ increased } & $\leq+4 \mathrm{mmol} / \mathrm{l}$ & $155(88 \%)$ & $283(61 \%)$ & $50(57 \%)$ & $86(73 \%)$ & $35(62 \%)$ & $47(65 \%)$ & $11(79 \%)$ & 53 \\
\hline & $>+7 \mathrm{mmol} / \mathrm{l}$ & $\begin{array}{l}1 \\
(0.5 \%)^{2,3,4,5,6,7}\end{array}$ & $86(19 \%)^{1}$ & $25(28 \%)^{1}$ & $16(14 \%)^{1}$ & $15(27 \%)^{1}$ & $18(25 \%)^{1}$ & $2(14 \%)^{1}$ & \\
\hline & $\leq+7 \mathrm{mmol} / \mathrm{l}$ & 190 (99.5\%) & $378(81 \%)$ & $63(72 \%)$ & $101(86 \%)$ & $41(73 \%)$ & $54(75 \%)$ & $12(86 \%)$ & 55 \\
\hline \multirow{2}{*}{$\begin{array}{l}\text { Rumen } \\
\text { chloride } \\
\text { (normal) }\end{array}$} & \multirow{2}{*}{$\begin{array}{l}6-30 \mathrm{mmol} / \mathrm{l} \\
<6 \mathrm{or}> \\
30 \mathrm{mmol} / \mathrm{l}\end{array}$} & \multirow{2}{*}{$\begin{array}{l}178 \\
(98 \%)^{2,3,4,5,6,7} \\
3(2 \%)\end{array}$} & $\begin{array}{l}375 \\
(87 \%)^{1,3,5}\end{array}$ & \multirow{2}{*}{$\begin{array}{l}51 \\
(64 \%)^{1,2}\end{array}$} & $80(75 \%)^{1}$ & \multirow{2}{*}{$\begin{array}{l}30 \\
(56 \%)^{1,2} \\
24(44 \%)\end{array}$} & \multirow{2}{*}{$\begin{array}{l}52(78 \%)^{1} \\
15(22 \%)\end{array}$} & \multirow{2}{*}{$\begin{array}{l}6(60 \%)^{1} \\
4(40 \%)\end{array}$} & \multirow[t]{2}{*}{94} \\
\hline & & & $58(13 \%)$ & & $27(25 \%)$ & & & & \\
\hline $\begin{array}{l}\text { Rumen } \\
\text { chloride }\end{array}$ & $>30 \mathrm{mmol} / \mathrm{l}$ & $3(2 \%)^{2,3,4,5,6,7}$ & $58(13 \%)^{1,3,5}$ & $\begin{array}{l}29 \\
(36 \%)^{1,2}\end{array}$ & $27(25 \%)^{1}$ & $\begin{array}{l}24 \\
(44 \%)^{1,2}\end{array}$ & $15(22 \%)^{1}$ & $4(40 \%)^{1}$ & \\
\hline \multirow[t]{3}{*}{ increased } & $\leq 30 \mathrm{mmol} / \mathrm{l}$ & 178 (98\%) & 375 (87\%) & $51(64 \%)$ & 80 (75\%) & 30 (56\%) & 52 (78\%) & $6(60 \%)$ & 94 \\
\hline & $>40 \mathrm{mmol} / \mathrm{l}$ & $1(0.5 \%)^{3,4,5,6}$ & $26(6 \%)^{3,5}$ & $\begin{array}{l}15 \\
(19 \%)^{1,2}\end{array}$ & $11(10 \%)^{1}$ & $\begin{array}{l}14 \\
(26 \%)^{1,2}\end{array}$ & $6(9 \%)^{1}$ & $1(10 \%)$ & \\
\hline & $\leq 40 \mathrm{mmol} / \mathrm{l}$ & $181(99.5 \%)$ & 407 (94\%) & $65(81 \%)$ & $96(90 \%)$ & $4074 \%)$ & $61(91 \%)$ & $9(90 \%)$ & 53 \\
\hline \multicolumn{10}{|c|}{${ }^{1}$ Different from controls $\mathrm{P}<0.01$} \\
\hline \multicolumn{10}{|c|}{${ }^{2}$ Different from TRP $P<0.01$} \\
\hline \multicolumn{10}{|c|}{${ }^{3}$ Different from U1 $\mathrm{P}<0.01$} \\
\hline \multicolumn{10}{|c|}{${ }^{4}$ Different from U2 $\mathrm{P}<0.01$} \\
\hline \multicolumn{10}{|c|}{${ }^{5}$ Different from U3 $P<0.01$} \\
\hline \multicolumn{10}{|c|}{${ }^{6}$ Different from U4 $P<0.01$} \\
\hline${ }^{7}$ Different fro & n U 5 P $<0.01$ & & & & & & & & \\
\hline
\end{tabular}

\section{Results}

\section{Haematocrit}

The median haematocrit ranged from 14 to $40 \%$ among all groups (Table 1). Cows with TRP and U2 had a significantly lower haematocrit (TRP $30 \%$, U2 14\%) and cows with U4 a significantly higher haematocrit (40\%) than controls (33\%). The percentage of cows with a haematocrit in the reference interval was $87 \%$ in controls, and was significantly lower in all other groups (Table 2, Fig. 1). Anaemia with haematocrits $<27$ and $<20 \%$ occurred in 4 and $0 \%$ of controls, respectively. Significantly more cows with TRP and U2 had a haematocrit < 27\% (TRP 17\%, U2 79\%) than controls, and there were 
significantly more cows with a haematocrit $<20 \%$ in the U2 group compared with all other groups $(70 \%$ vs $0-7 \%)$. The $L R_{+}$for a haematocrit $<27 \%$ and $\leq 20 \%$ in cows with U2 was 21 and 70 , respectively (Table 3 ). Haemoconcentration with a haematocrit > 37\% occurred in $9 \%$ of controls and significantly more often in cows with U1 (35\%), U3 (27\%), U4 (63\%) and U5 (50\%). A haematocrit > 44\% was seen in 33\% of cows with U4 (33\%) but not in controls (Table 2). The LR for a haematocrit $>44 \%$ in cows with U4 was 33 (Table 3 ).

Table 1

Laboratory variables in healthy control cows and cattle with traumatic reticuloperitonitis (TRP), abomasal ulcer type 1 (U1), type 2 (U2), type 3 (U3), type 4 (U4) and type 5 (U5) (medians, 25 and 75\% percentiles in brackets)

\begin{tabular}{|c|c|c|c|c|c|c|c|}
\hline Variable & Controls & TRP & U1 & $\mathrm{U} 2$ & U3 & U4 & U5 \\
\hline \multirow[t]{2}{*}{ Haematocrit (\%) } & $33^{2,4,6}$ & $30^{1,3,4,5,6}$ & $34^{2,4,6}$ & $14^{1,2,3,5,6,7}$ & $34^{2,4,6}$ & $40^{1,2,3,4,5}$ & $38^{4,6}$ \\
\hline & $(30-35)$ & $(27-33)$ & $(30-39)$ & $(11-23)$ & $(31-38)$ & $(33-48)$ & $(28-41)$ \\
\hline \multirow[t]{2}{*}{ Leukocytes $(/ \mu \mathrm{l})$} & $8000^{2,6}$ & $9400^{1,6}$ & 7800 & $9000^{6}$ & $8150^{6}$ & $6250^{1,2,4,5}$ & 6100 \\
\hline & $(6500-9400)$ & $(7400-11850)$ & (5900-10'200) & $\left(6200-11^{\prime} 600\right)$ & $(6375-10 ’ 375)$ & $(4475-9200)$ & $(5000-8150)$ \\
\hline \multirow[t]{2}{*}{ Total protein $(\mathrm{g} / \mathrm{l})$} & $78^{2,4,6,7}$ & $84^{1,4,5,6,7}$ & $78^{4,6,7}$ & $48^{1,2,3,5,6,7}$ & $76^{2,4,6,7}$ & $65^{1,2,3,4,5}$ & $66^{1,2,3,4,5}$ \\
\hline & $(72-84)$ & $(78-90)$ & $(72-87)$ & $(41-60)$ & $(70-82)$ & $(54-74)$ & $(60-71)$ \\
\hline \multirow[t]{2}{*}{ Fibrinogen (g/l) } & $5.0^{2,3,4,5,6}$ & $8 \cdot 0^{1,3,4,5,6,7}$ & $6.0^{1,2,4}$ & $4.0^{1,2,3,5,6,7}$ & $6.5^{1,2,4}$ & $6.0^{1,2,4}$ & $5.4^{2,4}$ \\
\hline & $(4.0-6.0)$ & $(6.0-10.0)$ & $(4.0-8.0)$ & $(3.0-6.0)$ & $(6.0-9.0)$ & $(4.8-8.0)$ & $(4.8-6.5)$ \\
\hline \multirow[t]{2}{*}{ Urea (mmol/l) } & $4.3^{3,4,5,6}$ & $4.0^{3,4,5,6}$ & $6.6^{1,2,4}$ & $10.1^{1,2,3,5,6}$ & $5.7^{1,2,4}$ & $7.3^{1,2,4}$ & \\
\hline & $(3.3-5.4)$ & $(3.0-5.3)$ & $(4.7-8.8)$ & $(8.0-12.4)$ & $(3.9-7.4)$ & $(5.0-11.4)$ & $(4.2-9.4)$ \\
\hline \multirow[t]{2}{*}{ Potassium (mmol/l) } & $4 \cdot 2^{2,3,4,5,6}$ & $3.6^{1}$ & $3.5^{1}$ & $3.4^{1}$ & $3.4^{1}$ & $3.6^{1}$ & 3.6 \\
\hline & $(4.0-4.4)$ & $(3.3-4.0)$ & $(3.1-4.0)$ & $(3.0-3.9)$ & $(2.9-4.0)$ & $(3.1-4.0)$ & $(3.2-4.2)$ \\
\hline \multirow[t]{2}{*}{ Chloride (mmol/l) } & $100^{4,6}$ & $100^{6}$ & $100^{6}$ & $103^{1,5,6}$ & $98^{4}$ & $96^{1,2,3,4}$ & 101 \\
\hline & $(97-103)$ & $(97-103)$ & $(93-106)$ & $(99-106)$ & $(92-103)$ & $(92-101)$ & $(95-104)$ \\
\hline Base excess & $1.7^{2}$ & $3.1^{1,4}$ & 3.6 & $0.3^{2}$ & 2.4 & 2.1 & 1.1 \\
\hline$(\mathrm{mmol} / \mathrm{l})$ & $(-0.4-3.3)$ & $(-0.3-5.7)$ & $(-1.0-7.9)$ & $(-3.1-4.2)$ & $(-1.2-8.2)$ & $(-2.0-6.9)$ & $(-1.2-3.9)$ \\
\hline Rumen chloride & $13^{2,3,4,5,6}$ & $20^{1,5}$ & $25^{1}$ & $22^{1,5}$ & $29^{1,2,4,6}$ & $22^{1,5}$ & 28 \\
\hline$(\mathrm{mmol} / \mathrm{l})$ & $(12-16)$ & $(15-26)$ & $(19-36)$ & $(18-31)$ & $(21-43)$ & $(17-29)$ & $(18-34)$ \\
\hline \multicolumn{8}{|c|}{${ }^{1}$ Difference to Controls $P<0.05$, Kruskal Wallis test } \\
\hline \multicolumn{8}{|c|}{${ }^{2}$ Difference to TRP $\mathrm{P}<0.05$, Kruskal Wallis test } \\
\hline \multicolumn{8}{|c|}{${ }^{3}$ Difference to U1 $\mathrm{P}<0.05$, Kruskal Wallis test } \\
\hline \multicolumn{8}{|c|}{${ }^{4}$ Difference to U2 $\mathrm{P}<0.05$, Kruskal Wallis test } \\
\hline \multicolumn{8}{|c|}{${ }^{5}$ Difference to U3 $\mathrm{P}<0.05$, Kruskal Wallis test } \\
\hline \multicolumn{8}{|c|}{${ }^{6}$ Difference to U4 $\mathrm{P}<0.05$, Kruskal Wallis test } \\
\hline${ }^{7}$ Difference to U5 P $<$ & 5 , Kruskal V & test & & & & & \\
\hline
\end{tabular}

Table 3.

Diagnostic sensitivity and specificity, positive and negative predictive values and positive likelihood ratio of laboratory variables in cows with traumatic reticuloperitonitis (TRP), abomasal ulcer type 1 (U1), type 2 (U2), type 3 (U3), type 4 (U4) and type 5 (U5) (in percent) 


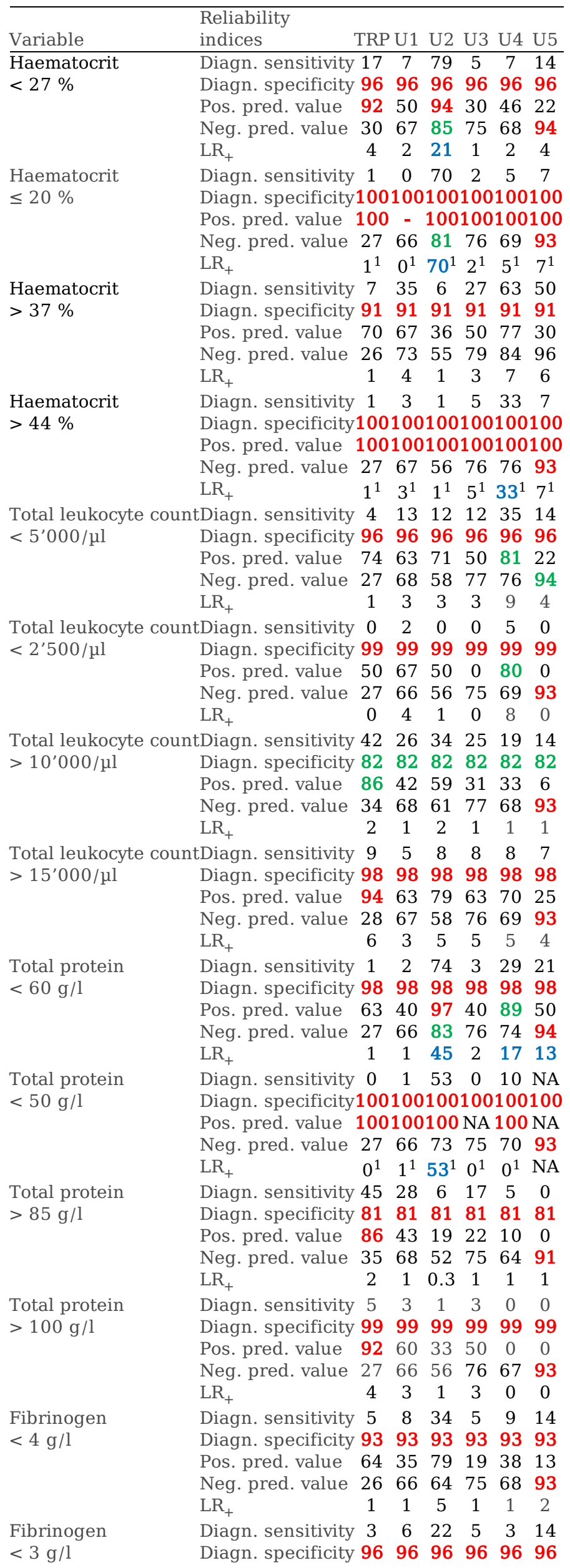

Page 9/21 
Fibrinogen
$>7 \mathrm{~g} / \mathrm{l}$

Fibrinogen $>9 \mathrm{~g} / \mathrm{l}$

Urea

$>6.5 \mathrm{mmol} / \mathrm{l}$

Urea

$>8.5 \mathrm{mmol} / \mathrm{l}$

Potassium

$<4 \mathrm{mmol} / \mathrm{l}$

Potassium

$<3 \mathrm{mmol} / \mathrm{l}$

Chloride

$\leq 90 \mathrm{mmol} / \mathrm{l}$

Chloride

$\leq 80 \mathrm{mmol} / \mathrm{l}$

Base excess

$<-4 \mathrm{mmol} / \mathrm{l}$

Base excess

$<-8 \mathrm{mmol} / \mathrm{l}$

Base excess

$>+4 \mathrm{mmol} / \mathrm{l}$

Base excess

$>+7 \mathrm{mmol} / \mathrm{l}$

Rumen chloride $>30 \mathrm{mmol} / \mathrm{l}$

Rumen chloride
Pos. pred. value $\begin{array}{lllllll}68 & 46 & 82 & 30 & 30 & 22\end{array}$ Neg. pred. value $\begin{array}{lllllll}27 & 67 & 61 & 75 & 68 & 94\end{array}$ $\begin{array}{lllllll}\mathrm{LR}_{+} & 1 & 2 & 6 & 1 & 1 & 4\end{array}$ Diagn. sensitivity $\begin{array}{llllll}69 & 29 & 8 & 43 & 45 & 21\end{array}$ Diagn. specificity $87 \quad 87 \quad 87 \quad 87 \quad 87 \quad 87$ Pos. pred. value $\begin{array}{llllll}93 & 53 & 33 & 52 & 62 & 11\end{array}$ Neg. pred. value $\begin{array}{llllll}51 & 71 & 55 & 82 & 77 & 93\end{array}$ $\begin{array}{lllllll}\mathrm{LR}_{+} & 5 & 2 & 1 & 3 & 3 & 2\end{array}$ Diagn. sensitivity $45 \quad 11 \quad 5 \quad 23 \quad 16 \quad 0$ Diagn. specificity $989898 \quad 98 \quad 98 \quad 98$ Pos. pred. value $\begin{array}{lllllll}99 & 77 & 70 & 82 & 82 & 0\end{array}$ Neg. pred. value $\begin{array}{lllllll}39 & 68 & 57 & 80 & 71 & 93\end{array}$ $\begin{array}{lllllll}\mathrm{LR}_{+} & 27 & 7 & 3 & 14 & 10 & 0\end{array}$ Diagn. sensitivity $14 \quad 51 \quad 89 \quad 35 \quad 56 \quad 43$ Diagn. specificity 949494949494 Pos. pred. value $\begin{array}{llllll}86 & 81 & 92 & 66 & 81 & 35\end{array}$ Neg. pred. value $\begin{array}{llllll}28 & 79 & 91 & 81 & 82 & 96\end{array}$ $\begin{array}{lllllll}\mathrm{LR}_{+} & 2 & 8 & 15 & 6 & 9 & 7\end{array}$ Diagn. sensitivity $6 \quad 28 \quad 70 \quad 13 \quad 41 \quad 29$ Diagn. specificity $99 \quad 99 \quad 99 \quad 99 \quad 99 \quad 99$ Pos. pred. value $979699 \quad 8997 \quad 80$ Neg. pred. value $28 \quad 73 \quad 81 \quad 78 \quad 78 \quad 95$ $\begin{array}{llllll}\mathrm{LR}_{+} & 115012824 & 54 & 52\end{array}$ Diagn. sensitivity $72 \quad 68 \quad 81 \quad 75 \quad 72 \quad 71$ Diagn. specificity $77 \quad 77 \quad 77 \quad 77 \quad 77 \quad 77$ Pos. pred. value $\begin{array}{llllll}90 & 60 & 73 & 52 & 60 & 19\end{array}$ Neg. pred. value $\begin{array}{lllllll}50 & 82 & 84 & 90 & 85 & 97\end{array}$ $\begin{array}{lllllll}\mathrm{LR}_{+} & 3 & 3 & 4 & 3 & 3 & 3\end{array}$

Diagn. sensitivity $13 \quad 21 \quad 20 \quad 25 \quad 20 \quad 14$ Diagn. specificity $99 \quad 99 \quad 99 \quad 99 \quad 99 \quad 99$ Pos. pred. value 989597949467 Neg. pred. value $\begin{array}{llllll}29 & 71 & 61 & 80 & 72 & 94\end{array}$ $\begin{array}{lllllll}\mathrm{LR}_{+} & 23 & 39 & 37 & 46 & 36 & 26\end{array}$ Diagn. sensitivity $7 \begin{array}{llllll}7 & 18 & 8 & 22 & 20 & 14\end{array}$ Diagn. specificity $98 \quad 98 \quad 98 \quad 98 \quad 98 \quad 98$ Pos. pred. value $\quad \begin{array}{llllll}90 & 81 & 73 & 76 & 81 & 33\end{array}$ Neg. pred. value $\begin{array}{lllllll}28 & 70 & 58 & 79 & 72 & 94\end{array}$ $\begin{array}{lllllll}\mathrm{LR}_{+} & 3 & 8 & 4 & 10 & 9 & 7\end{array}$ Diagn. sensitivity $2 \begin{array}{llllll}2 & 9 & 1 & 3 & 5 & 7\end{array}$ Diagn. specificity $99 \quad 99 \quad 99 \quad 99 \quad 99 \quad 99$ Pos. pred. value $\quad 80 \quad 80 \quad 50 \quad 50 \quad 67 \quad 33$ Neg. pred. value $27 \quad \begin{array}{lllllll}68 & 56 & 76 & 69 & 93\end{array}$ $\begin{array}{lllllll}\mathrm{LR}_{+} & 1 & 8 & 1 & 3 & 4 & 7\end{array}$ Diagn. sensitivity $6 \quad 11 \quad 20 \quad 5 \quad 17 \quad 14$ Diagn. specificity 959595959595 Pos. pred. value $\begin{array}{llllll}74 & 53 & 72 & 25 & 57 & 18\end{array}$ Neg. pred. value $\begin{array}{lllllll}29 & 70 & 66 & 77 & 75 & 94\end{array}$ $\begin{array}{lllllll}\mathrm{LR}_{+} & 1 & 2 & 4 & 1 & 4 & 3\end{array}$ Diagn. sensitivity $00 \begin{array}{lllll}0 & 11 & 0 & 6 & 7\end{array}$ Diagn. specificity $99 \quad 99 \quad 99 \quad 99 \quad 99 \quad 99$ Pos. pred. value $\begin{array}{llllll}67 & 75 & 93 & 0 & 80 & 50\end{array}$ Neg. pred. value $29 \quad 6965 \quad 77 \quad 74 \quad 94$ $\begin{array}{lllllll}\mathrm{LR}_{+} & 1 & 7 & 21 & 0 & 11 & 14\end{array}$ Diagn. sensitivity $\begin{array}{llllll}39 & 42 & 26 & 36 & 35 & 21\end{array}$ Diagn. specificity $88 \quad 88 \quad 88 \quad 88 \quad 88 \quad 88$ Pos. pred. value $\begin{array}{lllllll}89 & 62 & 57 & 47 & 52 & 12\end{array}$ Neg. pred. value $\begin{array}{lllllll}37 & 77 & 66 & 82 & 78 & 94\end{array}$ $\begin{array}{lllllll}\mathrm{LR}_{+} & 3 & 3 & 2 & 3 & 3 & 2\end{array}$ Diagn. sensitivity $1928 \quad 14 \quad 27 \quad 25 \quad 14$ Diagn. specificity $99 \quad 99 \quad 99 \quad 99 \quad 99 \quad 99$ Pos. pred. value 999694949567 Neg. pred. value $\begin{array}{llllll}33 & 75 & 65 & 82 & 78 & 94\end{array}$ $\begin{array}{lllllll}\mathrm{LR}_{+} & 35 & 54 & 26 & 51 & 48 & 27\end{array}$ Diagn. sensitivity $13 \quad 36 \quad 25 \quad 44 \quad 22 \quad 40$ Diagn. specificity 989898989898 Pos. pred. value $\begin{array}{lllllll}95 & 91 & 90 & 89 & 83 & 57\end{array}$ Neg. pred. value $\begin{array}{llllll}32 & 78 & 69 & 86 & 77 & 97\end{array}$ $\begin{array}{lllllll}\mathrm{LR}_{+} & 8 & 22 & 15 & 27 & 14 & 24\end{array}$ Diagn. sensitivity $6 \begin{array}{llllll}6 & 10 & 26 & 9 & 10\end{array}$ 
> $40 \mathrm{mmol} / \mathrm{l} \quad$ Diagn. specificity 999999999999

Pos. pred. value 969492938650

Neg. pred. value 317465827595

$\begin{array}{lllllll}\mathrm{LR}_{+} & 11 & 34 & 19 & 47 & 16 & 18\end{array}$

1 Calculation of the $\mathrm{LR}_{+}$required the reduction of the diagnostic specificity from $100 \%$ to $99 \%$

NA, not applicable

Values between 80 and $89 \%$ are in green

Values between 90 and 100\% are in red

Values $\geq 10$ are in blue

\section{Total leukocyte count}

The median total leukocyte count ranged from 6,100 to 9,400 cells/ $\mu$ l among all groups (Table 1 ). The total leukocyte count was significantly higher $(9,400$ cells $/ \mu \mathrm{l})$ in cows with TRP and significantly lower $(6,250 \mathrm{cells} / \mu \mathrm{l})$ in cows with U4 compared with controls $(8,000 / \mu \mathrm{l})$. The total leukocyte count was within in the reference interval in $78 \%$ (Table 2, Fig. 2), and leukopenia with $<5,000$ and $<2,500$ leukocytes/ $\mu$ l was seen in 4 and $1 \%$ of controls, respectively (Table 2). Leukopenia was significantly more common in cows with U4 (35\%) than in controls (5\%), cows with TRP (4\%), U1 (13\%), U2 (12\%) and U3 (12\%). The LR for leukopenia was low (0-9; Table 3).

\section{Total protein concentration}

The median total protein concentration ranged from 48 to $84 \mathrm{~g} / \mathrm{l}$ (Table 1 ) and was significantly higher in cows with TRP (84 g /I) and significantly lower in cows with U2 (48 g/l), U4 (65 g/l) and U5 (66 g/l) compared with controls $(78 \mathrm{~g} / \mathrm{l})$. Seventy-nine percent of controls had a total protein concentration in the reference interval (Table 2, Fig. 3), 2\% had hypoproteinaemia and 19\% had hyperproteinaemia. Cows with U2 (75\%), U4 (29\%) and U5 (21\%) had hypoproteinaemia with < $60 \mathrm{~g} / \mathrm{l}$ significantly more often than controls ( $2 \%)$, and cows with U2 (53\%) and U4 (10\%) had total protein concentrations $<50 \mathrm{~g} / \mathrm{l}$ significantly more often than controls $(0 \%)$; concentrations $<50 \mathrm{~g} / \mathrm{l}$ were also significantly more common in cows with U2 compared with all other groups. The LR for hypoproteinaemia (<60 g/l) was 45, 17 and 13 in cows with U2, U4 and U5, respectively (Table 3). Hyperproteinaemia > $85 \mathrm{~g} / \mathrm{I}$ was significantly more common in cows with TRP (45\%) and significantly less common in cows with U2 (6\%) and U4 (5\%) than in controls (19\%). Total protein concentrations $>85$ and $>100 \mathrm{~g} / \mathrm{l}$ had positive predictive values of 86 and $92 \%$, respectively, for TRP (Table 3)

\section{Fibrinogen concentration}

The median fibrinogen concentration ranged from 4 to $8 \mathrm{~g} / \mathrm{l}$ (Table 1). The fibrinogen concentration was significantly higher in cows with TRP $(8.0 \mathrm{~g} / \mathrm{l}), \mathrm{U} 1(6.0 \mathrm{~g} / \mathrm{l}), \mathrm{U} 3(6.5 \mathrm{~g} / \mathrm{l})$ and U4 $(6.0 \mathrm{~g} / \mathrm{l})$ and significantly lower in cows with $\mathrm{U} 2(4.0 \mathrm{~g} / \mathrm{l})$ compared with controls (5.0 g/l). Eighty percent of control cows had a fibrinogen concentration in the reference interval (Table 2, Fig. 4), $7 \%$ had hypofibrinogenaemia and $13 \%$ had hyperfibrinogenaemia. Cows with U2 had fibrinogen concentrations $<4(34 \%)$ and $<3 \mathrm{~g} / \mathrm{I}(22 \%)$ significantly more often than controls. The LR $\mathrm{f}_{+}$for these test results ranged from 1 to 6 (Table 3). Cows with TRP (69\%), U1 (29\%), U3 (43\%) and U4 (45\%) had fibrinogen concentrations > $7 \mathrm{~g} / \mathrm{l}$ significantly more often than controls (13\%). Cows with TRP also had fibrinogen concentrations $>7$ and $>9 \mathrm{~g} / \mathrm{l}$ significantly more often than all other groups. The $\mathrm{LR}_{+}$for a fibrinogen concentration $>9 \mathrm{~g} / \mathrm{I}$ was 27 for TRP, 14 for U3 and 10 for U4 (Table 3).

\section{Urea concentration}

The median urea concentration ranged from 4.0 to $10.1 \mathrm{mmol} / \mathrm{I}$ (Table 1). Cows with U1 (6.6 mmol/I), U2 (10.1 mmol/I), U3 (5.7 mmol/I) and U4 $(7.3 \mathrm{mmol} / \mathrm{l})$ had significantly higher median urea concentrations than controls $(4.3 \mathrm{mmol} / \mathrm{l})$. Ninety-four percent of controls had a urea concentration in the reference interval (2.0-6.5 mmol/I), and 6\% had azotaemia (Table 2, Fig. 5). Cows with U1 (51 and $28 \%$ ), U2 (89 and $70 \%)$, U3 (35 and 13\%), U4 (56 and 41\%) and U5 (43 and 29\%) had urea concentrations $>6.5$ and $>8.5 \mathrm{mmol} / \mathrm{l}$, respectively, significantly more often than controls ( 6 and $0.5 \%)$. Cows with $\mathrm{U} 2$ had azotaemia $(89 \%>6.5$ and $70 \%>8.5 \mathrm{mmol} / \mathrm{l})$ significantly more often than cows of any other group. The LR for a urea concentration $>8.5 \mathrm{mmol} / \mathrm{I}$ was $>10$ in all groups and reached 128 and 74 for U2 and U4, respectively (Table 3 ).

\section{Potassium concentration}

The median potassium concentration ranged from 3.4 to $4.2 \mathrm{mmol} / \mathrm{I}$ (Table 1). Except for cows with U5, all cows from the other disease groups had significantly lower potassium concentrations than controls. Seventy-six percent of controls had a potassium concentration in the reference interval (Table 2, Fig. 6) and 23 and $0.5 \%$ had hypokalaemia with concentrations of $<4$ and $<3$ mmol/l, respectively. In cows of all disease groups, concentrations $<4$ ( 68 to $81 \%$ of cows) and $<3 \mathrm{mmol} / \mathrm{I}$ ( 13 to $25 \%$ of cows) were significantly more common than in controls. The LR for a potassium concentration $<3 \mathrm{mmol} / \mathrm{l}$ ranged from 23 to 46 in all disease groups (Table 3 ).

\section{Chloride concentration}


The median chloride concentration ranged from 96 to $103 \mathrm{mmol} / \mathrm{l}$ (Table 1). Eighty-nine percent of controls had normochloraemia (Table 2, Fig. 7) and 2 and $1 \%$ had hypochloraemia with concentrations $\leq 90$ and $\leq 80 \mathrm{mmol} / \mathrm{l}$, respectively. Cows with U1 (18\%), U3 (22\%) and U4 (20\%) had hypochloraemia significantly more often than controls, and cows with U1 had concentrations $\leq 80 \mathrm{mmol} / \mathrm{I}$ significantly more often ( $9 \%$ ) than controls. The $\mathrm{LR}_{+}$for a chloride concentration $\leq 90 \mathrm{mmol} / \mathrm{I}$ was 10 in cows with U3 (Table 3). Hyperchloraemia was significantly more common in cows with U1 (30\%) and U2 (28\%) than in controls.

\section{Base excess}

The median base excess ranged from 0.3 to $3.6 \mathrm{mmol} / \mathrm{I}$ (Table 1). Eighty-two percent of controls had normal values of -4 to $+4 \mathrm{mmol} / \mathrm{l}$ (Table 2 , Fig. 8), $6 \%$ had values lower and $12 \%$ had values greater than the reference interval. A base excess smaller than -4 and -8 mmol/I was recorded in 6 and $0.5 \%$ of controls, respectively (Table 2 ); values lower than $-4 \mathrm{mmol} / \mathrm{I}$ were significantly more common in cows with U2 (21\%) and U4 (18\%), and values lower than $-8 \mathrm{mmol} / \mathrm{I}$ were significantly more frequent in cows with U2 (11\%) than in controls. The LR for a base excess lower than $8 \mathrm{mmol} / \mathrm{I}$ was 21,11 and 14 in cows with $\mathrm{U} 2, \mathrm{U} 4$ and U5, respectively (Table 3 ). A positive base excess greater than +4 and $+7 \mathrm{mmol} / \mathrm{I}$ was significantly more frequent in cows with TRP, U1, U2, U3 and U4 (greater than $+4 \mathrm{mmol} / \mathrm{l}$ in 27 to $43 \%$; greater than $+7 \mathrm{mmol} / \mathrm{l}$ in 14 to $28 \%$ including cows with U5) than in controls. The LR for a base excess greater than $+7 \mathrm{mmol} / \mathrm{l}$ ranged from 26 to 54 for all groups (Table 3 ).

\section{Rumen chloride}

The median rumen chloride concentration ranged from 13 to $29 \mathrm{mmol} / \mathrm{l}$ (Table 1). Except for cows with U5, all disease groups had significantly higher rumen chloride concentrations than controls. Ninety-eight percent of controls had rumen chloride concentrations in the reference interval (Table 2, Fig. 9) and $2 \%$ had concentrations exceeding $30 \mathrm{mmol} / \mathrm{l}$. The percentage of cows from all disease groups with concentrations $>30 \mathrm{mmol} / \mathrm{l}$ (13 to $44 \%$ ) was significantly greater compared with controls. Except for TRP, the $L_{+}$for a rumen chloride concentration $>30 \mathrm{mmol} / \mathrm{I}$ was greater than 10 and ranged from 14 to 27 (Table 3).

\section{Total protein concentration and haematocrit}

The combined analysis of total protein concentration and haematocrit showed that $79 \%$ of cows with U 2 had a decreased haematocrit, which was accompanied by hypoproteinaemia (30-59 g/l) in 73\% and normoproteinaemia in 6\% (Fig. 10A). In the other disease groups, a decreased haematocrit occurred in no more than $17 \%$ of the cows and was accompanied by normo-, hypo- or hyperproteinaemia. Most cows with a normal haematocrit had normoproteinaemia, some had hyperproteinaemia whereas hypoproteinaemia was rare (Fig. 10B). Most cows with increased haematocrit had normoproteinaemia and only rarely hypo- or hyperproteinaemia (Fig. 10C).

\section{Total protein and fibrinogen concentrations}

Seventy-five percent of cows with U2 had hypoproteinaemia, which was accompanied by normofibrinogenaemia in $42 \%$, hypofibrinogenaemia in $32 \%$ and hyperfibrinogenaemia in $1 \%$ (Fig. 11A). Of the cows with normoproteinaemia (Fig. 11B), normofibrinogenaemia was most common in controls and cows with U1, U2, U3 and U5, and hyperfibrinogenaemia was most common in cows with TRP and U4. Most of the controls and cows with U1 with hyperproteinaemia had normofibrinogenaemia and most of the cows with TRP, U2, U3 and U4 with hyperproteinaemia had hyperfibrinogenaemia (Fig. 11C).

\section{Discussion}

Various studies have described the sensitivity, specificity and predictive value of total protein [19], total protein and fibrinogen [20] and fibrinogen, serum amyloid A and haptoglobin concentrations [21] for the diagnosis of cows with TRP; however, the results were compared with findings in ill cows and not healthy controls. Therefore, the calculated diagnostic parameters differ greatly from ours and a direct comparison is not possible. The laboratory variables used for comparison of healthy cows and cows with TRP, U1, U2, U3, U4 and U5 were selected from a comprehensive panel that included haematological, serum biochemical, blood gas and rumen fluid analyses and urinalysis. For a variable to be included in the study, it had to have been measured in the majority of cows and lie outside the reference interval in a large number of ill cows. Interestingly, numerous variables were outside the reference intervals in healthy cows including rumen chloride ( $2 \%)$, urea (6\%), serum chloride (11\%), haematocrit (13\%), base excess (18\%), fibrinogen $(20 \%)$, total protein $(21 \%)$, total leukocyte count $(22 \%)$ and potassium $(24 \%)$. This was despite the fact that the reference intervals of several variables had been modified and expanded. According to Constable and co-workers [22], healthy animals are assumed to have values within a certain range referred to as normal range or reference interval, and ill animals are assumed to have values outside this interval. A reference interval is established by using the results of a large number of healthy animals and usually includes $95 \%$ of the animals. Calculation of a reference interval is straightforward when the values have a normal distribution but requires transformation of non-normal data. In the present study, the variables had non-normal distributions with the exception of total protein and urea concentrations. Therefore, calculation of the reference interval assuming a normal distribution (mean \pm 2 standard deviations) would result in an exaggerated interval that would include a considerable number of ill animals, erroneously considered healthy. When reference intervals published in the veterinary literature were used to assess healthy cows, the following values were outside the reference interval: haematocrit (41\%), total protein (40\%), serum chloride (26\%), base excess (58\%) and rumen chloride (64\%). Therefore, the reference intervals of some variables were slightly expanded upward (total protein), downward (serum chloride and rumen chloride) or upward and downward (haematocrit, base excess). When healthy cows have values outside the reference intervals, they may be incorrectly diagnosed as ill (false positive). This problem can be mitigated by considering a combination of variables when making a diagnosis or to disregard certain values below the reference interval [22]. The extent of the deviation from the reference interval should also be considered because small deviations are less likely to reflect a disease than large ones. This can be quantified using the LR $R_{+}$, which expresses the association between a 
test result and the presence of a target disease [22]. The $\mathrm{LR}_{+}$expresses how much more likely an individual with the disease is to have a positive test result than if the individual is disease-free [23]. The $L_{+}$was calculated for all variables in the present study because it is an overall measure of the efficiency of a diagnostic test; it combines the diagnostic sensitivity and specificity and is not affected by the prevalence of the disease [22, 23]. The latter characteristic was of particular importance in the present study because the exact prevalence of TRP and abomasal ulcers is not known. Another drawback of using reference intervals is that not all diseased animals have values outside the interval. When a single variable with a value within the reference interval is used to make a diagnosis, a sick animal may be erroneously identified as not having the disease (false negative). This can be mitigated by shrinking the reference interval but in doing so the rate of false-positives increases. It would be better to consider groups of variables that all have diagnostic utility for the disorder in question. This was done in the present study by combining haematocrit and total protein, and total protein and fibrinogen concentrations.

Rumen chloride was the best variable for the differentiation of healthy and diseased cows because only $2 \%$ of controls had values outside the reference interval. This was also supported by an $\mathrm{LR}_{+}$of 14 to 27 in cows with ulcers. An $L R_{+}$of 14 means that the likelihood of increased rumen chloride in an ill cow is 14 times more likely to occur than in a cow without an ulcer. The usefulness of rumen chloride for differentiating the different disease groups was limited. Only cows with TRP had rumen chloride concentrations $<40 \mathrm{mmol} / \mathrm{I}$ significantly less often (6\%) than cows with U1 and U3. From a practical standpoint, this means that TRP is unlikely in a cow with a marked increase in rumen chloride concentration.

Although of slightly less importance than rumen chloride, urea had a high diagnostic specificity and was well suited for differentiation of healthy and diseased cows. The urea concentration was $>6.5 \mathrm{mmol} / \mathrm{l}$ in only $6 \%$ and $>8.5 \mathrm{mmol} / \mathrm{l}$ in only $0.5 \%$ of controls, and the $L R_{+}$for a urea concentration $>$ $8.5 \mathrm{mmol} / \mathrm{I}$ ranged from 11 in cows with TRP to 128 in cows with U2. Except for cows with TRP, azotaemia was significantly more frequent in ill cows than in controls. Cows with U2 (70\%) had urea concentrations $>8.5$ significantly more often than cows of the other groups, which may have been prerenal azotaemia attributable to hypovolaemia. Analogous to increased rumen chloride, an increase in urea concentration (above $8.5 \mathrm{mmol} / \mathrm{l}$ ) is rare $(6 \%)$ in cows with TRP because this is a localised disease with no direct systemic effects.

Even though $13 \%$ of controls had a haematocrit outside the expanded reference interval of 27 to $37 \%$, none had a haematocrit $>44 \%$. Likewise, no more than $5 \%$ of cows with TRP, U1, U2 and U3 had values $>44 \%$, which was in contrast to cows with U4, in which $33 \%$ had a haematocrit $>44 \%$. Thus, in cows with a high haematocrit, TRP or type 1, 2, 3 or 5 abomasal ulcers are unlikely. A haematocrit $\leq 20 \%$ occurred in $70 \%$ of cows with U2 and in only 0 to $7 \%$ of cows in the other groups and thus allowed differentiation of cows with U2.

Leukocytosis was a poor criterion for differentiating healthy and diseased cows and among cows of the different disease groups. The utility of leukopenia for this purpose was similar except that a total leukocyte count $<5000 / \mu$ l was seen in $35 \%$ of cows with U4 but in only 4 to $14 \%$ of cows of the other groups. Persistent leukopenia is always a serious finding. Leukopenia in cows with U4 can be interpreted as a sequel to sepsis and leukocyte consumption [14]. Bone marrow production of leukocytes is insufficient in the face of severe inflammation because mobilisation of the marrow stem cell pool is slower in cattle than in other species [24].

A potassium concentration $<3 \mathrm{mmol} / \mathrm{l}$ is almost always a sign of disease because it occurred in only $0.5 \%$ of controls. However, hypokalaemia was non-specific and all disease groups were affected. The principal cause of hypokalaemia is decreased forage intake associated with illness [25].

A total protein concentration > $85 \mathrm{~g} / \mathrm{l}$ was measured in $45 \%$ cows with TRP, which was significantly more frequent than in cows of the other groups. Similar observations were made for fibrinogen concentrations $>9 \mathrm{~g} / \mathrm{l}$. Diagnostic accuracy can be improved by considering multiple laboratory variables; $36 \%$ of cows with TRP had both hyperproteinaemia and fibrinogenaemia (Fig. 11C), which was in agreement with the results of previous reports [19-21]. Conversely, 75\% of cows with U2 had hypoproteinaemia ( $<60 \mathrm{~g} / \mathrm{I})$ and $32 \%$ had hypofibrinogenaemia (< $4 \mathrm{~g} / \mathrm{l}$, Fig. $11 \mathrm{~B})$. Cows with U4 also had hypoproteinaemia (<60 g/l) significantly more frequently than cows of the other groups but only $4 \%$ had concurrent hypofibrinogenaemia (Fig. 11A). Interestingly, 79\% of cows with U2 had anaemia accompanied by hypoproteinaemia in $73 \%$ (Fig. $10 \mathrm{~A}$ ), and $63 \%$ of cows with U4 had an increased haematocrit accompanied by normoproteinaemia in most cases (Fig. 10C).

\section{Conclusions}

Depending on the variable, 2 to $24 \%$ of healthy cows had values outside the reference intervals, which made the differentiation of healthy and diseased cows difficult based on laboratory variables alone. Rumen chloride and urea concentrations provided the best selectivity because they were outside the reference intervals in only 2 and $6 \%$ of healthy cows, respectively. Although there were significant differences between groups of cows with respect to several laboratory variables, there were no variables that reliably differentiated cows with different diseases. Instead of using a specific laboratory variable for diagnosis, a combination of multiple variables must be considered along with the history and clinical findings and often the results of additional techniques such as ultrasonography.

\section{Abbreviations}

TRP: Traumatic reticuloperitonitis; U1: Abomasal ulcer type-1; U2: Abomasal ulcer type-2; U3: Abomasal ulcer type-3; U4: Abomasal ulcer type-4; U5: Abomasal ulcer type-5; LR : Positive likelihood ratio; Fig: Figure. 


\section{Declarations}

\section{Ethics approval and consent to participate}

The study was not submitted to our institutional ethics committee, because it was a retrospective study using medical records of cows that had been referred to our clinic for examination and treatment over a time period of 14 years $(2001-2014)$. No experimental animals were used. Under these circumstances, ethical approval is not required in Switzerland. Written consent was obtained from all owners to participate.

\section{Consent for publication}

All owners signed a consent form allowing us to use the animals and all the associated medical data for scientific analysis and publication.

\section{Availability of data and materials}

The datasets used and analysed for this study are available from the corresponding author on reasonable request.

\section{Competing interests}

The authors declare that they have no competing interests.

\section{Fundings}

Not applicable since it was a retrospective analysis of medical records.

\section{Author's contributions}

UB initiated, planned and supervised the study and prepared the manuscript, SW and CR analysed the medical histories of the cows as part of her dissertation, KN and CG made contributions to acquisition and interpretation of data and were involved in drafting the manuscript. All authors read and approved the final manuscript.

\section{Acknowledgements}

The authors thank the agricultural assistants for their help with the clinical examinations.

\section{Author details}

Department of Farm Animals, Vetsuisse-Faculty, University of Zurich, Winterthurerstrasse 260, CH-8057 Zurich, Switzerland. Vetsuisse-Faculty, University of Zurich,

\section{References}

1. Dirksen G. Krankheiten von Haube und Pansen beim ruminanten Rind. In: Dirksen G, Gründer HD, Stöber M, editors. Innere Medizin und Chirurgie des Rindes. Berlin: Parey Buchverlag; 2002. p. 396-455.

2. Constable PD, Hinchcliff KW, Done SH, Grünberg W. Diseases of the alimentary tract - ruminant. In: Constable PD, Hinchcliff KW, Done SH, Grünberg W, editors. Veterinary Medicine. A Textbook of the Diseases of Cattle, Horses, Sheep, Pigs, and Goats. St. Louis, Missouri: Elsevier; 2017. p. 436-621.

3. Whitlock RH. Bovine stomach diseases. In: Anderson NV, editor. Veterinary Gastroenterology. Philadelphia: Lea and Febiger; 1980. p. 425-8.

4. Smith DF, Munson L, Erb HN. Abomasal ulcer disease in adult dairy cattle. Cornell Vet. 1983;73:213-24.

5. Constable PD. Abomasal ulcers. In: Line S, editor. The Merck Veterinary Manual, 10th. edn., New York: Merck and Co., Inc.; 2010. p. 219-22.

6. Hund A, Wittek T. Abomasal and third compartment ulcers in ruminants and south american camelids. Vet Clin North Am Food Anim Pract. 2018;34:35-54.

7. Braun U, Reif C, Hilbe M, Gerspach C. Type-5 abomasal ulcer and omental bursitis in 14 cows. Acta Vet Scand. 2020;62:4.

8. Braun U, Eicher R, Ehrensperger F. Type 1 abomasal ulcers in dairy cattle. J Vet Med A. 1991;38:357-66.

9. Munch SL, Nielsen SS, Krogh MA, Capion N. Prevalence of abomasal lesions in Danish Holstein cows at the time of slaughter. J Dairy Sci. 2019;102:5403-9.

10. Braun U, Warislohner S, Torgerson P, Nuss K, Gerspach C. Clinical and laboratory findings in 503 cattle with traumatic reticuloperitonitis. BMC Vet Res. 2018;14:66.

11. Braun U, Gerspach C, Reif C, Hilbe M, Nuss K. Clinical, laboratory and ultrasonographic findings in 94 cows with type-1 abomasal ulcer. Schweiz Arch Tierheilk. 2020;162:235-44. 
12. Braun U, Gerspach C, Nuss K, Hässig M, Hilbe M, Reif C. Clinical and laboratory findings, treatment and outcome in 145 cows with type-2 abomasal ulcer. Res Vet Sci. 2019;124:366-74.

13. Braun U, Gerspach C, Hilbe M, Devaux DJ, Reif C. Clinical and laboratory findings in 60 cows with type-3 abomasal ulcer. Schweiz Arch Tierheilk. 2019;161:523-31.

14. Braun U, Reif C, Nuss K, Hilbe M, Gerspach C. Clinical, laboratory and ultrasonographic findings in 87 cows with type-4 abomasal ulcer. BMC Vet Res. 2019;15:100.

15. Braun U, Nuss K, Warislohner S, Reif C, Oschlies C, Gerspach C. Frequency and diagnostic reliability of clinical signs in cows with traumatic reticuloperitonitis and abomasal ulcers. BMC Vet Res. 2020;16:359.

16. 16. Braun U, Amrein E, Estermann U, Egli J, Schweizer T, Lutz H, Ehrensperger F, Vandevelde M, Kihm Untersuchungen an 182 Nachkommen von an boviner spongiformer Enzephalopathie (BSE) erkrankten Kühen in der Schweiz. Teil 1: Klinische Befunde. Schweiz Arch Tierheilk. 1998;140:240-9.

17. Braun U, Beckmann C, Gerspach C, Hässig M, Muggli E, Knubben-Schweizer G, Nuss K. Clinical findings and treatment in cattle with caecal dilatation. BMC Vet Res. 2012;8:75.

18. Dirksen G. Pansensaftentnahme und -untersuchung. In: Dirksen G, Gründer HD, Stöber M, editors. Gustav Rosenberger. Die klinische Untersuchung des Rindes. Berlin, Hamburg: Paul Parey; 1990. p. 312-26.

19. Dubensky RA, White ME. The sensitivity, specificity and predictive value of total plasma protein in the diagnosis of traumatic reticuloperitonitis. Can J Comp Med. 1983; 47:241-4.

20. Jafarzadeh SR, Nowrouzian I, Khaki Z, Ghamsari SM, Adibhashemi F. The sensitivities and specificities of total plasma protein and plasma fibrinogen for the diagnosis of traumatic reticuloperitonitis in cattle. Prev Vet Med. 2004;65:1-7.

21. Nazifi S, Ansari-Lari M, Asadi-Fardqi J, Rezaei M. The use of receiver operating characteristic (ROC) analysis to assess the diagnostic value of serum amyloid A, haptoglobin and fibrinogen in traumatic reticuloperitonitis in cattle. Ve. J. 2009;182:315-9.

22. Constable PD, Hinchcliff KW, Done SH, Grünberg W. Interpretation of laboratory data. In: Constable PD, Hinchcliff KW, Done SH, Grünberg W, editors. Veterinary Medicine. A Textbook of the Diseases of Cattle, Horses, Sheep, Pigs, and Goats. St. Louis, Missouri: Elsevier; 2017. p. $22-5$.

23. Petrie A, Watson P. Additional techniques. In: Petrie A, Watson P, editors. Statistics for Veterinary and Animal Science. Chichester, West Sussex: Wiley-Blackwell; 2013. p. 200-29.

24. Tornquist SJ, Rigas S. Interpretation of ruminant leukocyte responses. In: Weiss DJ, Wardrop KJ, editors. Schalm's Veterinary Hematology. Ameslowa: Wiley-Blackwell; 2010. p. 307-13.

25. Sattler N, Fecteau G. Hypokalemia syndrome in cattle. Vet Clin North Am Food Anim Pract. 2014;30:351-7.

\section{Figures}

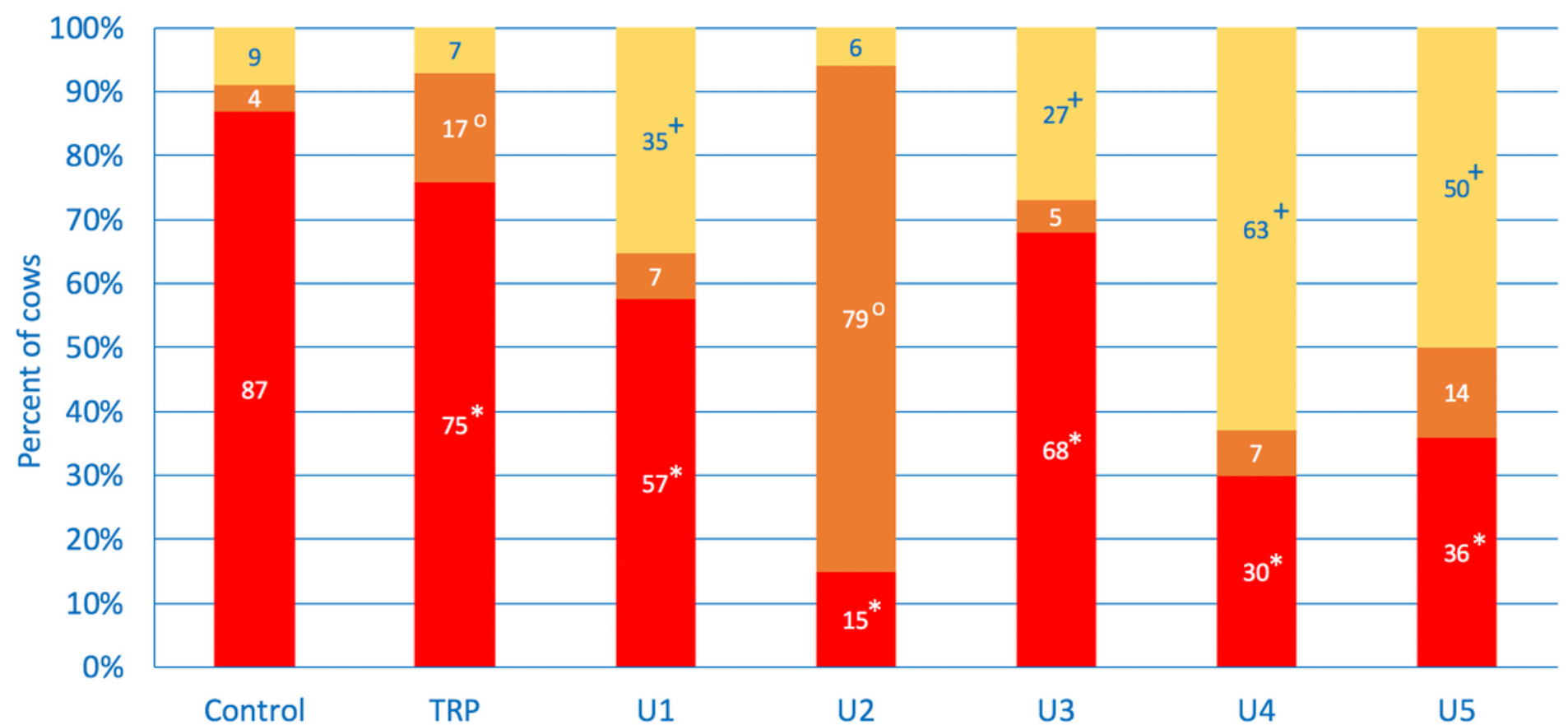


Figure 1

Frequency distribution of normal, decreased and increased haematocrit values in control cows and in cows with traumatic reticuloperitonitis (TRP) and type 1 (U1), type 2 (U2), type 3 (U3), type 4 (U4) and type 5 (U5) abomasal ulcer. * , o, + within classifications, percentages differ from controls (P $<0.05$ )

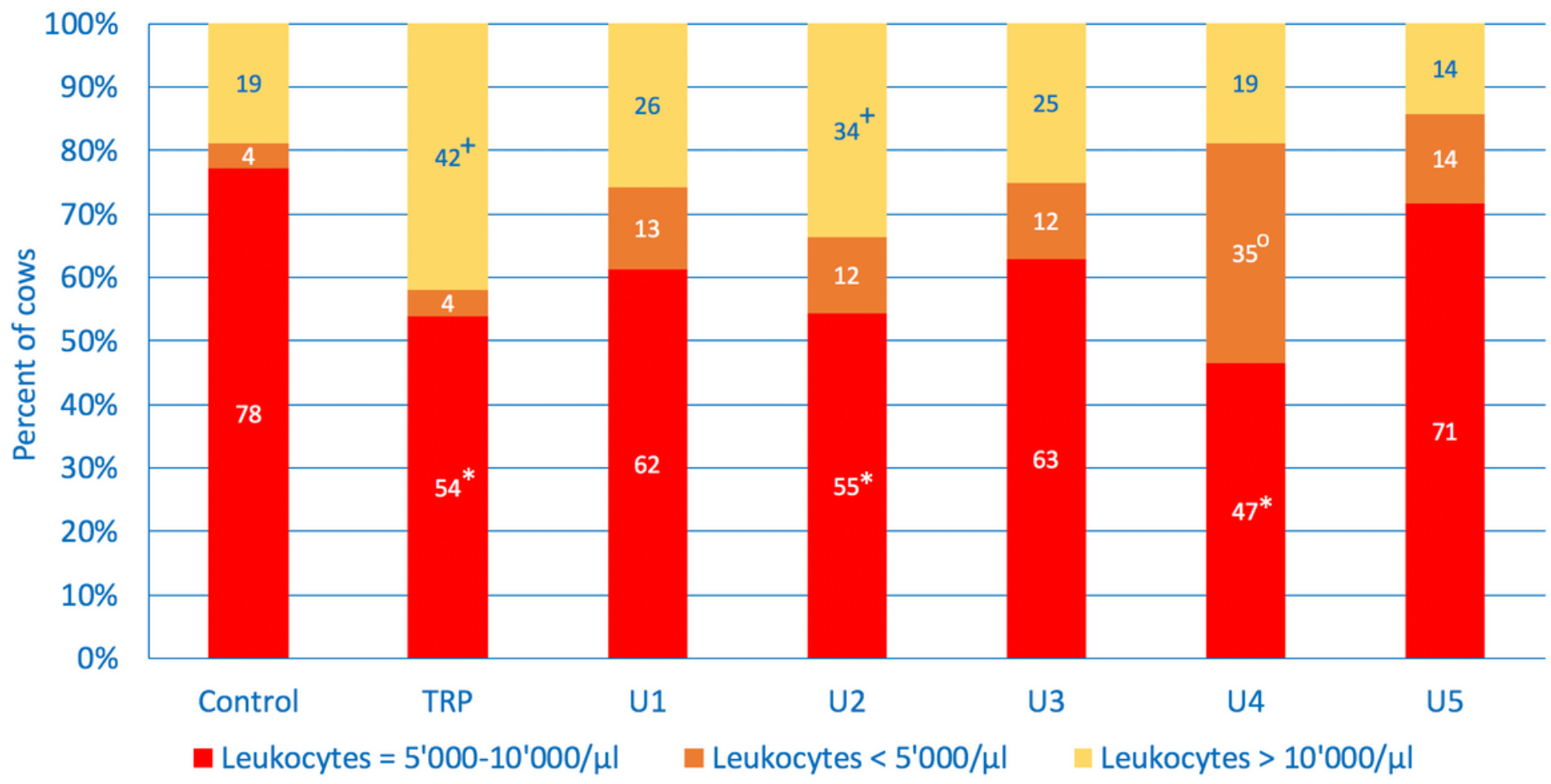

\section{Figure 2}

Frequency distribution of normal, decreased and increased total leukocyte counts in control cows and in cows with TRP, U1, U2, U3, U4 and U5. For key see Fig. 1

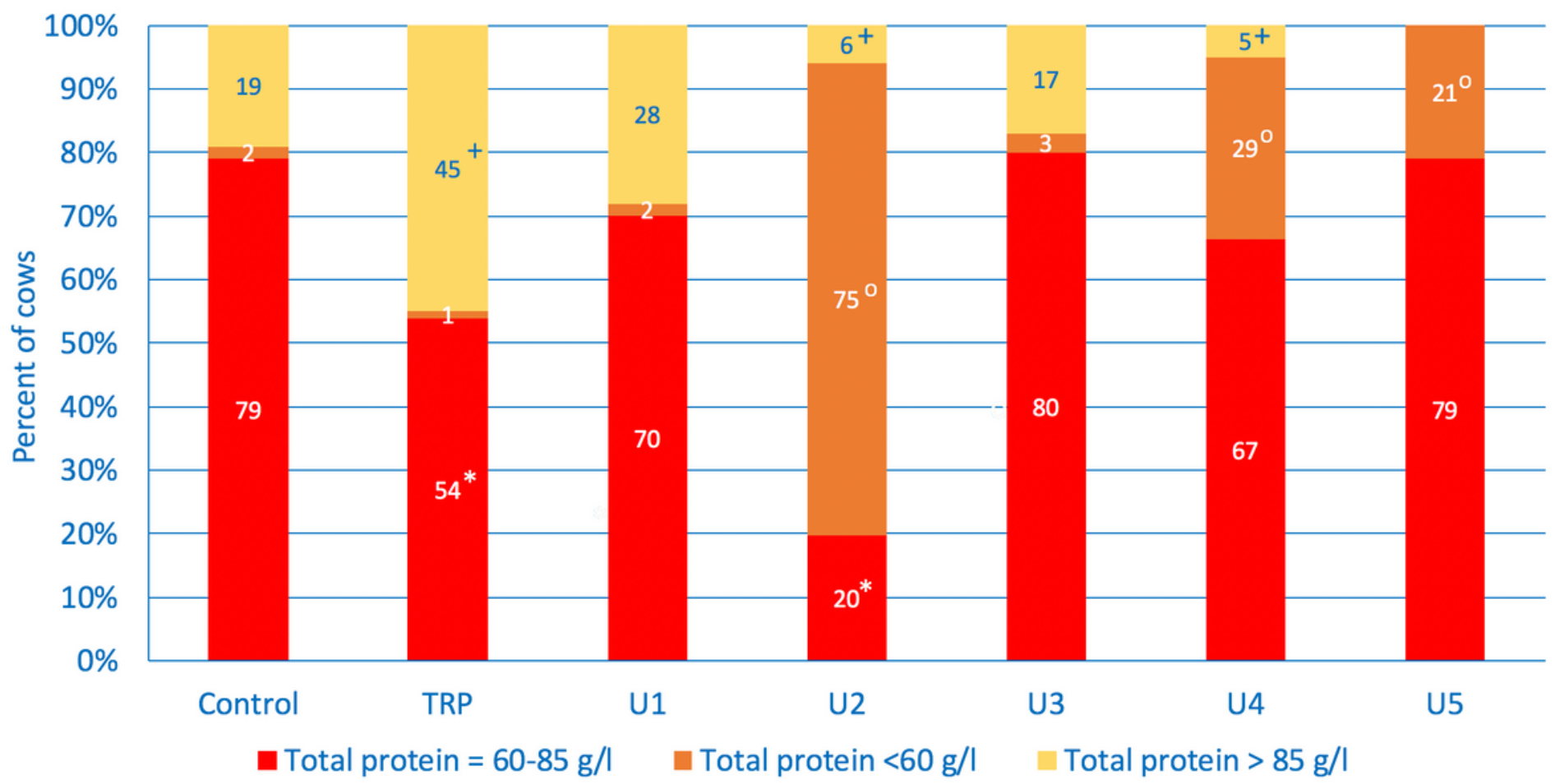

Figure 3 
Frequency distribution of normal, decreased and increased total protein concentrations in control cows and in cows with TRP, U1, U2, U3, U4 and U5. For key see Fig. 1

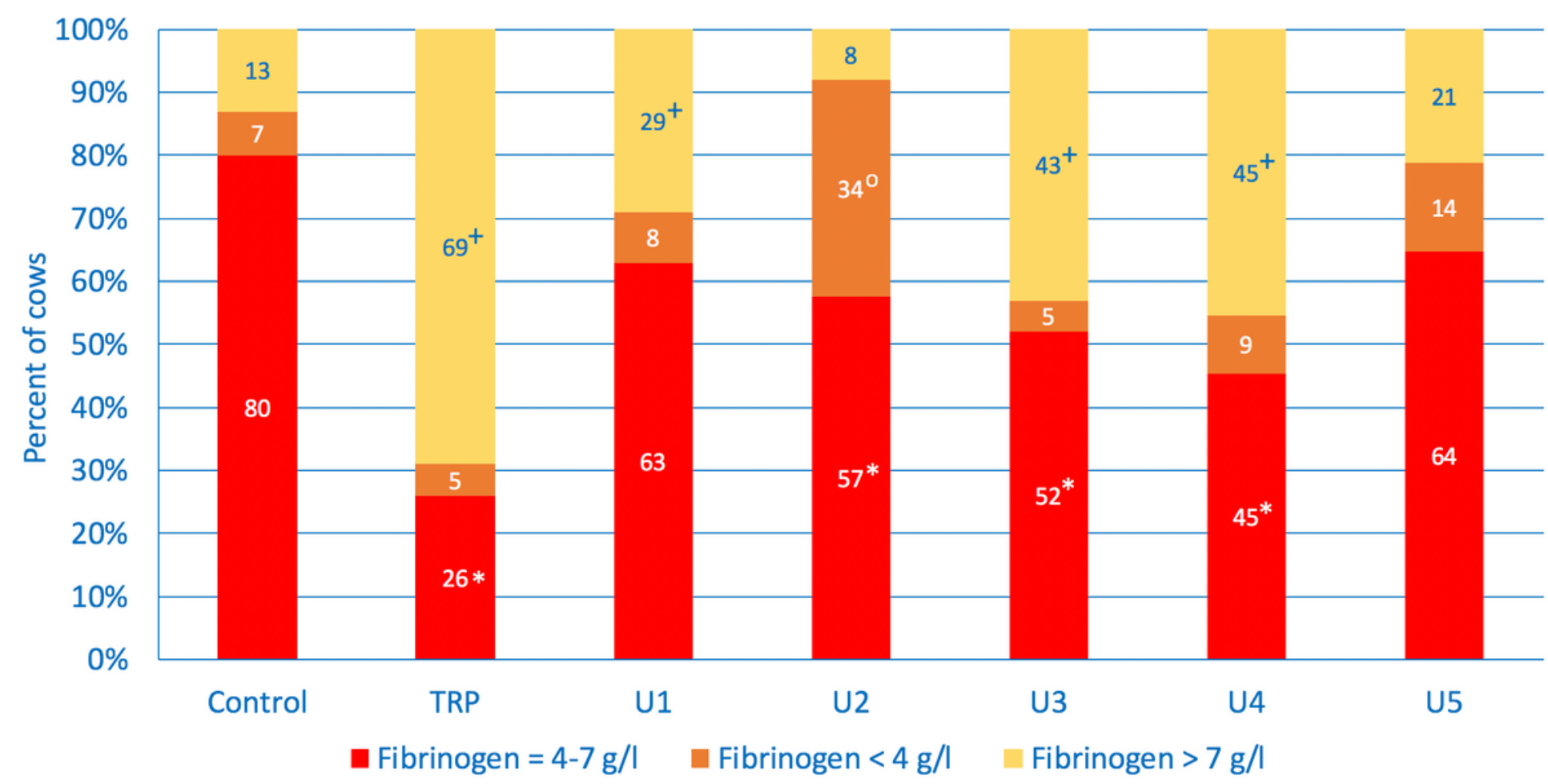

\section{Figure 4}

Frequency distribution of normal, decreased and increased fibrinogen concentrations in control cows and in cows with TRP, U1, U2, U3, U4 and U5. For key see Fig. 1

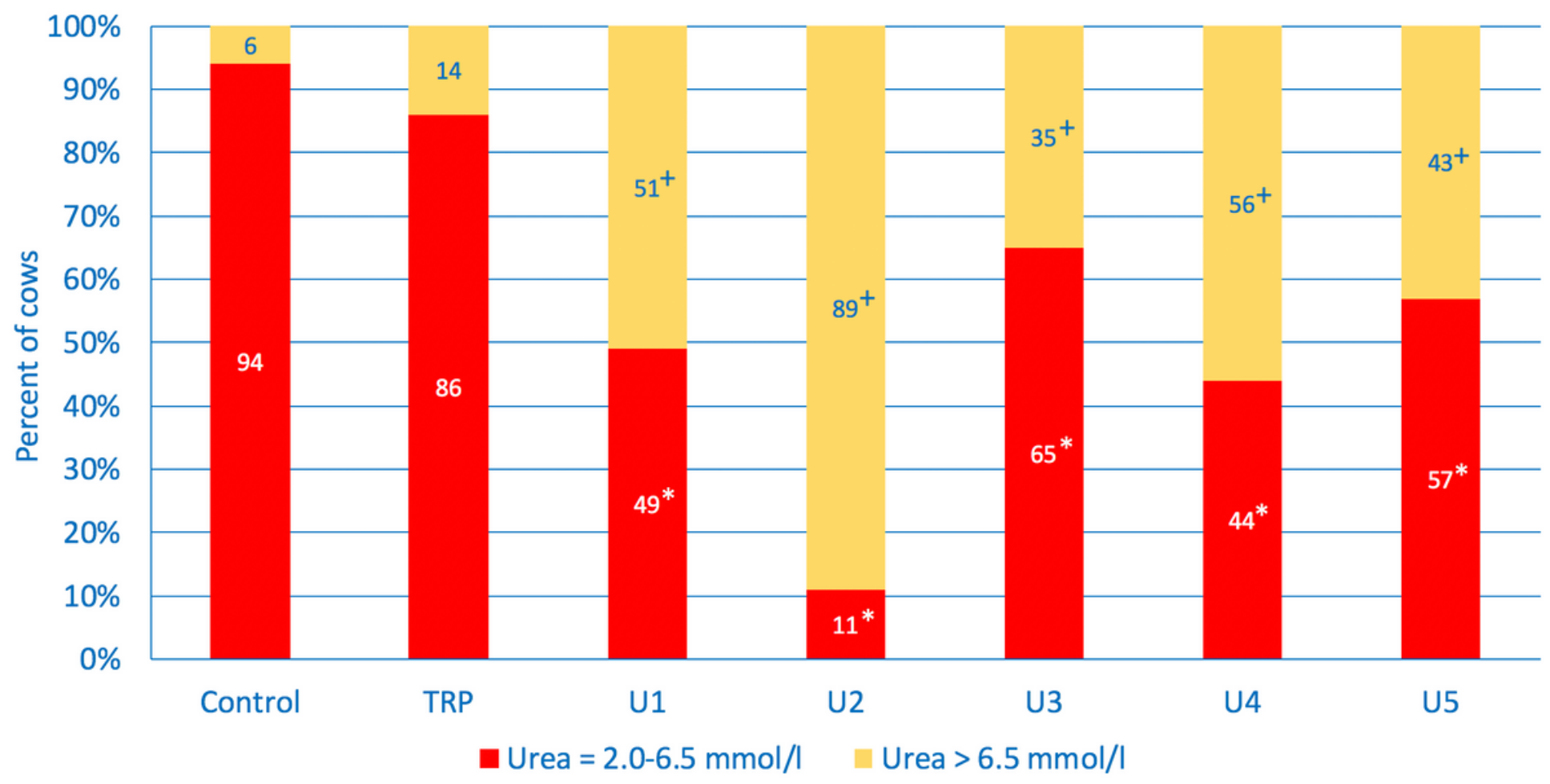

\section{Figure 5}

Frequency distribution of normal, decreased and increased urea concentration in control cows and in cows with TRP, U1, U2, U3, U4 and U5. For key see Fig. 1 


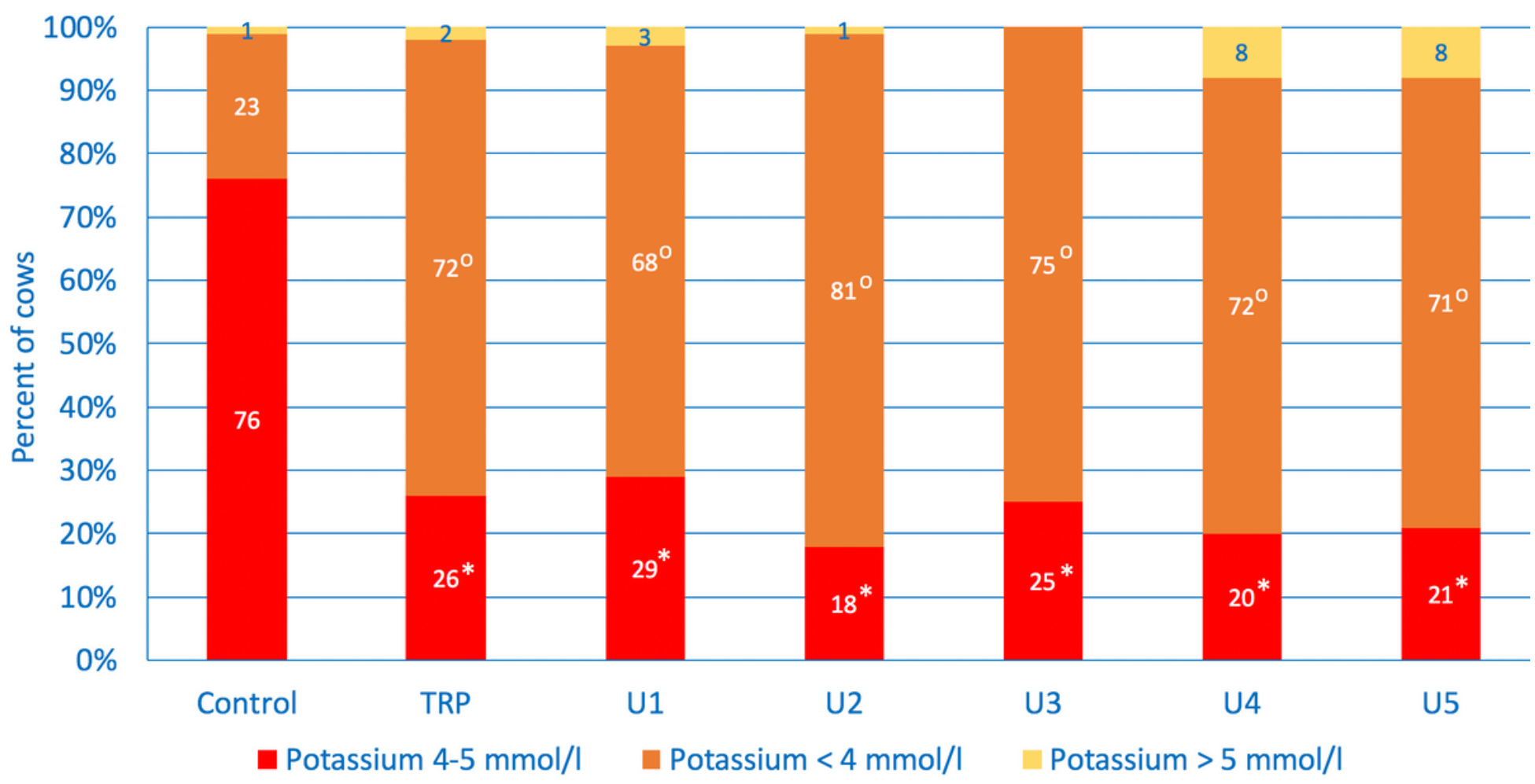

\section{Figure 6}

Frequency distribution of normal, decreased and increased potassium concentrations in control cows and in cows with TRP, U1, U2, U3, U4 and U5. For key see Fig. 1

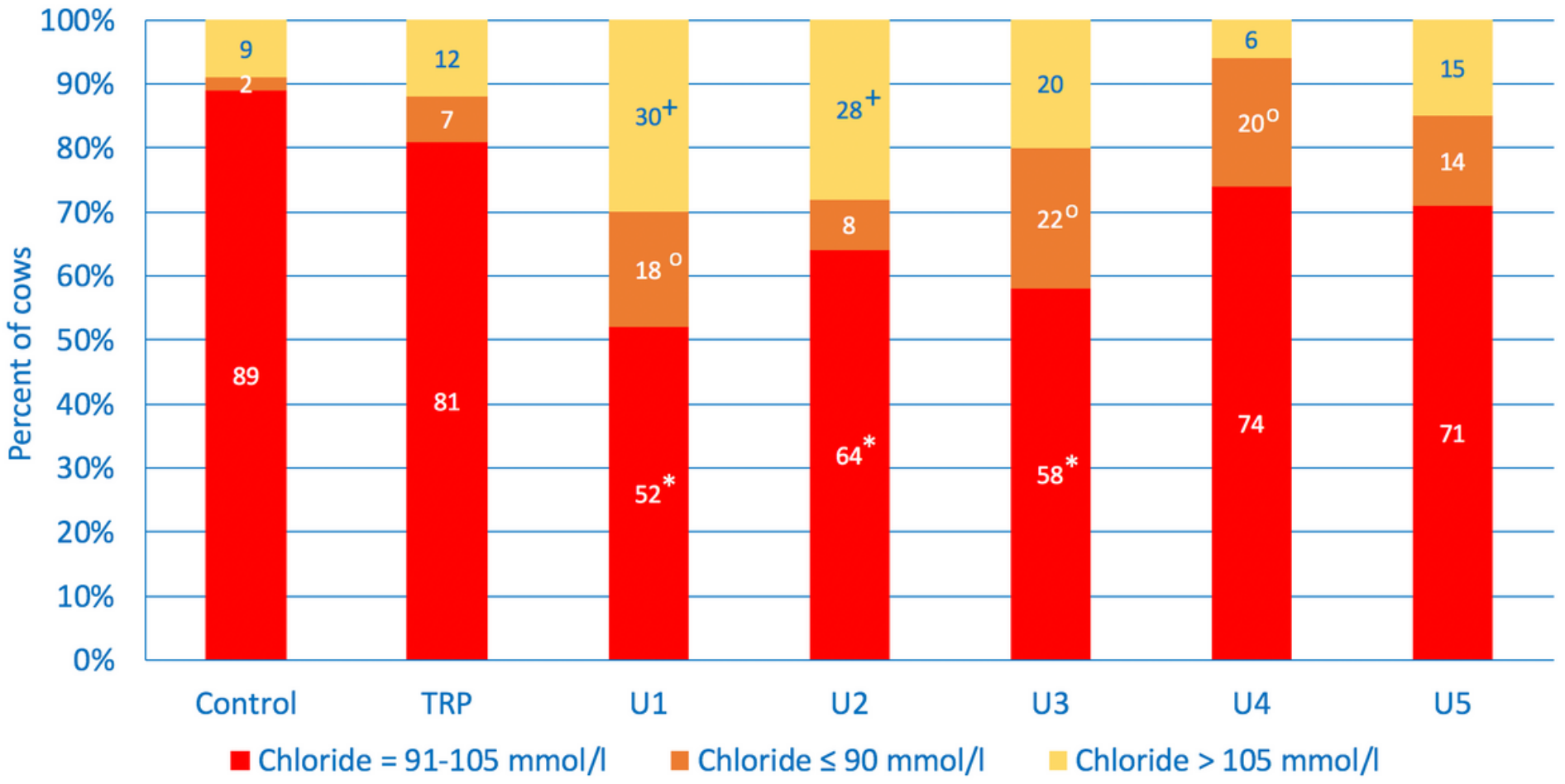

\section{Figure 7}

Frequency distribution of normal, decreased and increased chloride concentrations in control cows and in cows with TRP, U1, U2, U3, U4 and U5. For key see Fig. 1 


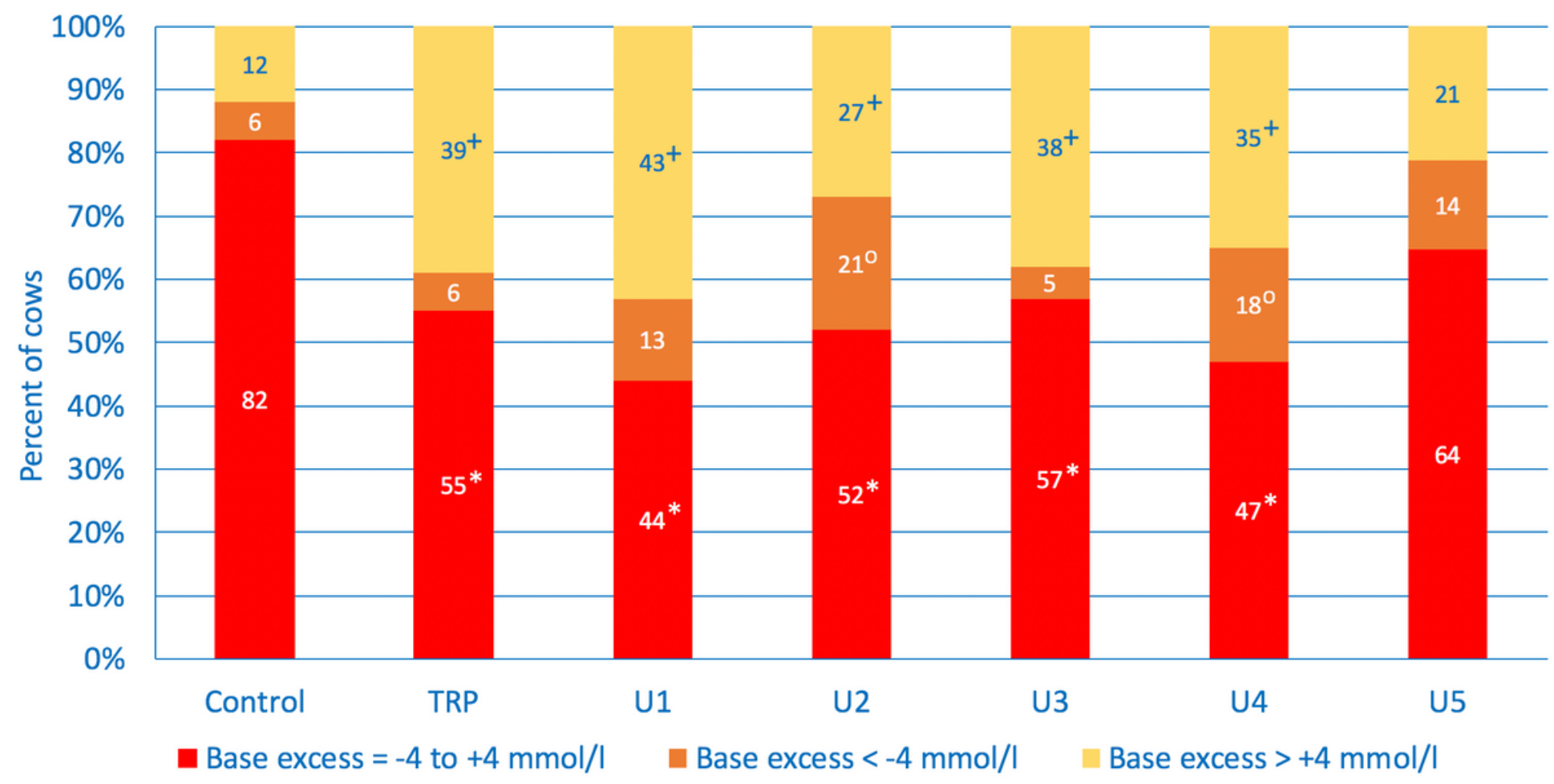

\section{Figure 8}

Frequency distribution of normal, decreased and increased base excess in control cows and in cows with TRP, U1, U2, U3, U4 and U5. For key see Fig. 1

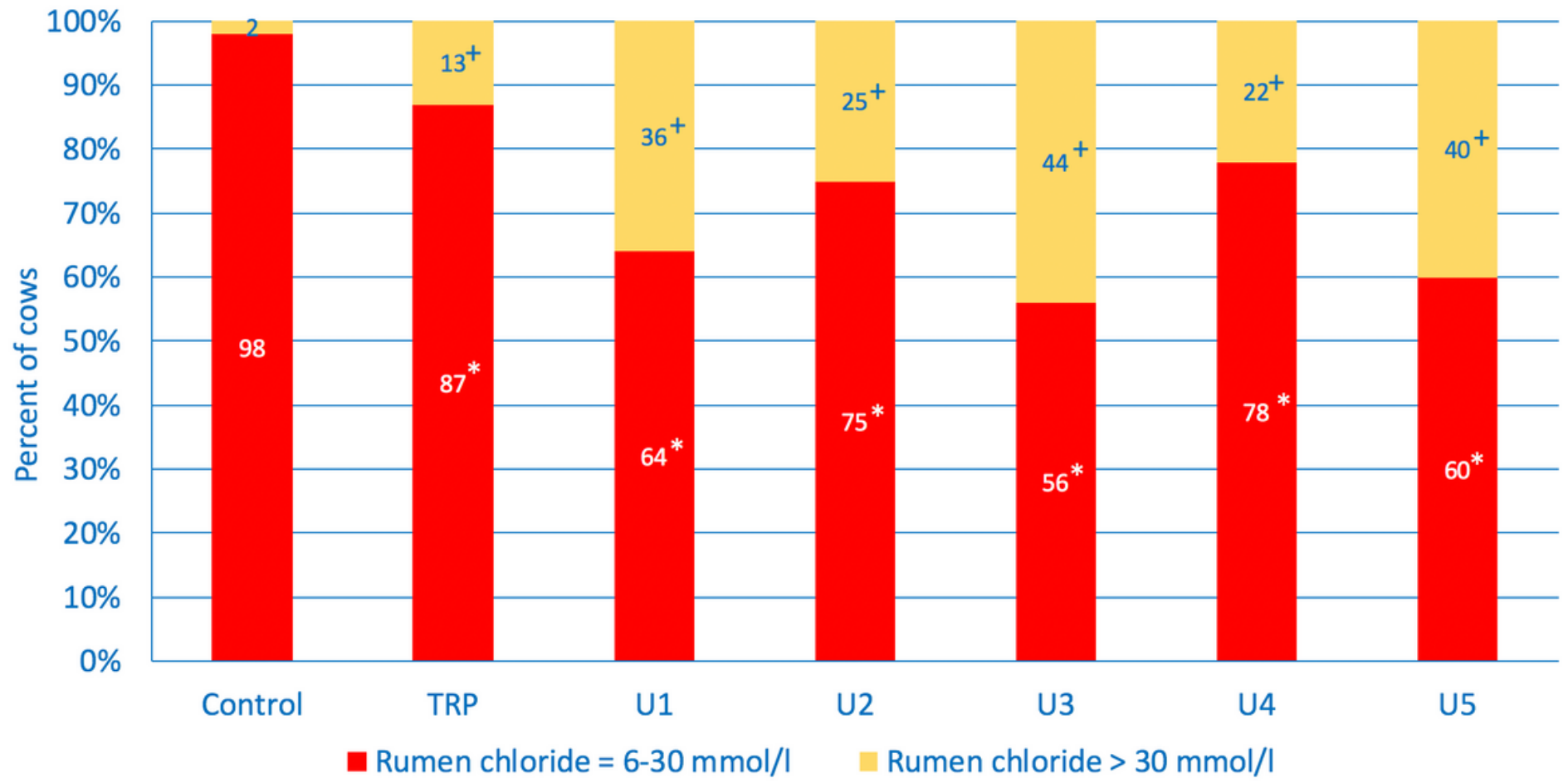

\section{Figure 9}

Frequency distribution of normal, decreased and increased rumen chloride concentrations in control cows and in cows with TRP, U1, U2, U3, U4 and U5. For key see Fig. 1 

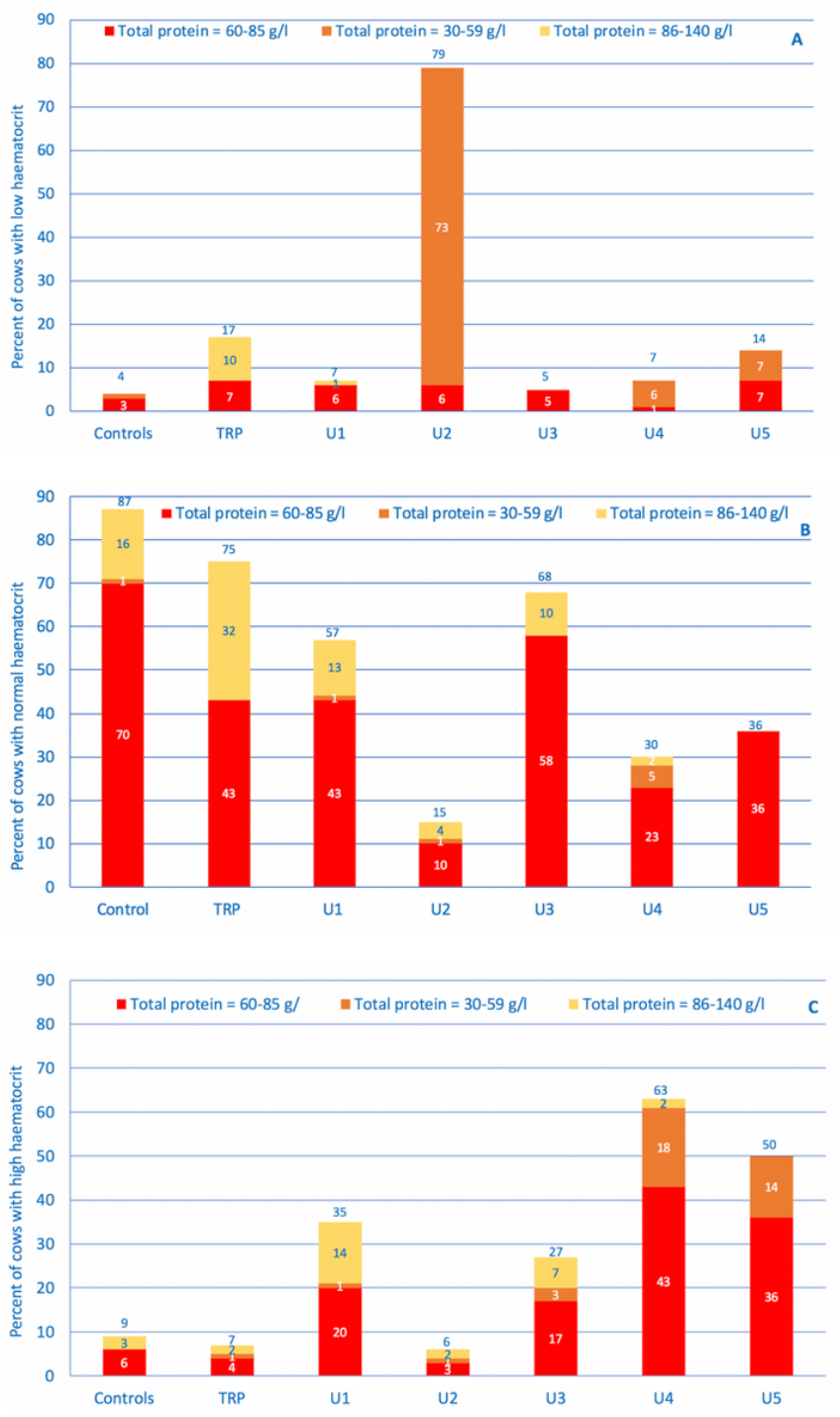

Figure 10

Frequency distribution of total protein concentrations in cows with decreased $(<27 \%, A)$, normal $(27-37 \%$, B) and increased haematocrit values $(>$ $37 \%, \mathrm{C})$ in control cows and cows with TRP, U1, U2, U3, U4 and U5. For key see Fig. 1 

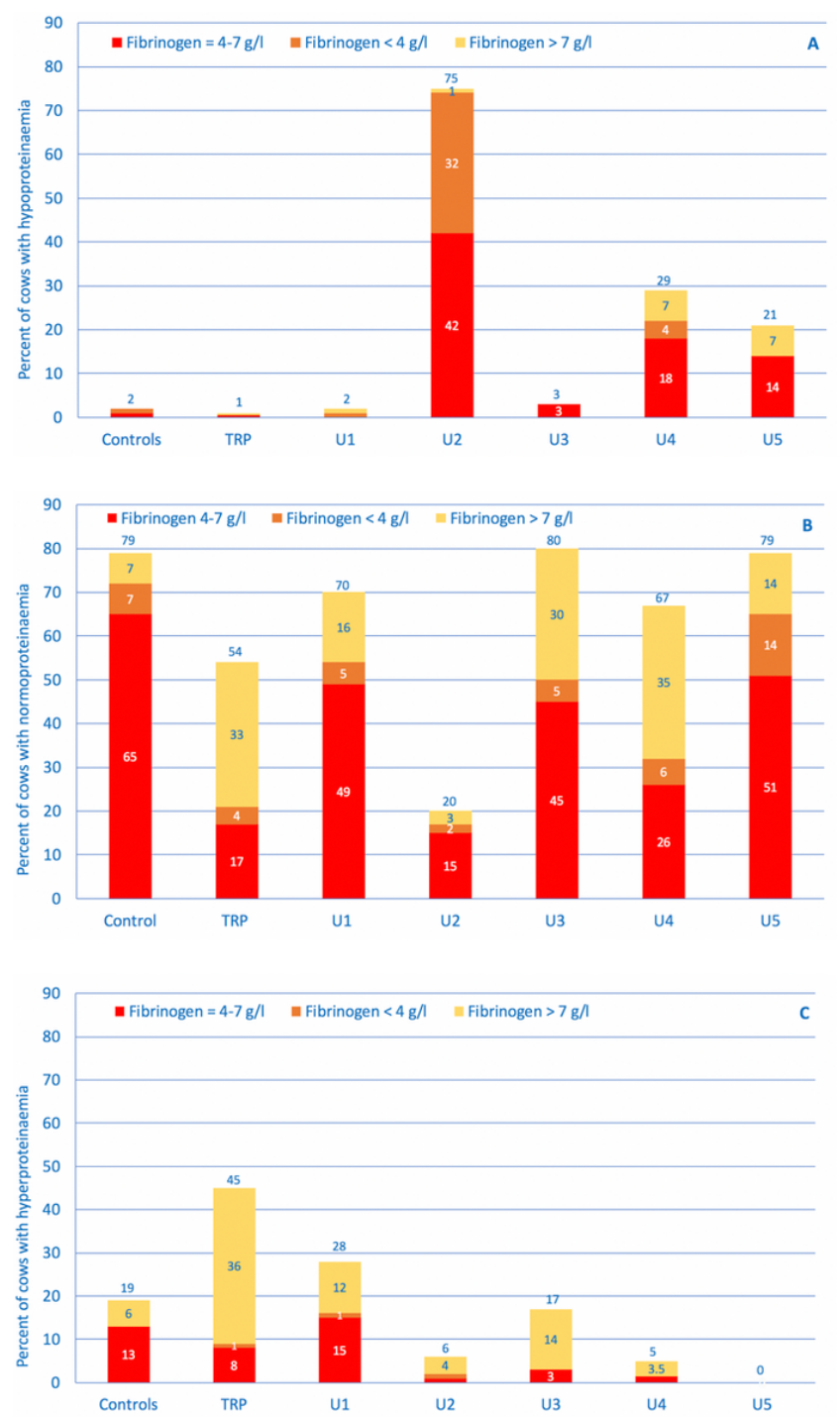

Figure 11

Frequency distribution of fibrinogen concentrations in cows with decreased ( $<60 \mathrm{~g} / \mathrm{l}, \mathrm{A})$, normal $(60-85 \mathrm{~g} / \mathrm{l}, \mathrm{B})$ and increased total protein concentration (> $85 \mathrm{~g} / \mathrm{l}, \mathrm{C}$ ) in control cows and cows with TRP, U1, U2, U3, U4 and U5. For key see Fig. 1

\section{Supplementary Files}

This is a list of supplementary files associated with this preprint. Click to download.

- Letter2.docx

- Letter.docx 